\title{
Temporal Entropy Generation in the Viscous Layers of Laterally-Converging Duct Flows
}

Donald M. McEligot Robert S. Brodkey Helmut Eckelmann

December 2008

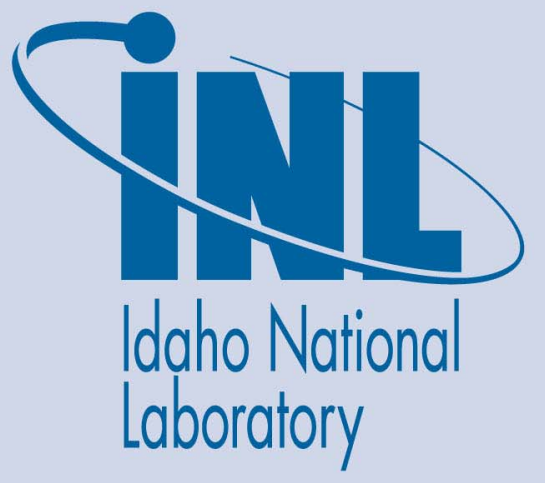

The INL is a U.S. Department of Energy National Laboratory operated by Battelle Energy Alliance 


\title{
Temporal Entropy Generation in the Viscous Layers of Laterally-Converging Duct Flows
}

${ }^{1}$ Ohio State University

${ }^{2}$ Universität Göttingen
Donald M. McEligot

Robert S. Brodkey ${ }^{1}$

Helmut Eckelmann ${ }^{2}$

December 2008

\author{
Idaho National Laboratory \\ Nuclear Science and Technology \\ Idaho Falls, Idaho 83415
}

http://www.inl.gov

Prepared for the

U.S. Department of Energy

Office of Nuclear Energy

Under DOE Idaho Operations Office

Contract DE-AC07-05ID14517 


\section{DISCLAIMER}

This information was prepared as an account of work sponsored by an agency of the U.S. Government. Neither the U.S. Government nor any agency thereof, nor any of their employees, makes any warranty, expressed or implied, or assumes any legal liability or responsibility for the accuracy, completeness, or usefulness, of any information, apparatus, product, or process disclosed, or represents that its use would not infringe privately owned rights. References herein to any specific commercial product, process, or service by trade name, trade mark, manufacturer, or otherwise, does not necessarily constitute or imply its endorsement, recommendation, or favoring by the U.S. Government or any agency thereof. The views and opinions of authors expressed herein do not necessarily state or reflect those of the U.S. Government or any agency thereof. 



\begin{abstract}
Since insight into entropy generation is a key to increasing efficiency and thereby reducing fuel consumption and/or waste and -- for wall-bounded flows -most entropy is generated in the viscous layer, we examine the transient behavior of its dominant contributor there for a non-canonical flow. New measurements in oil flow are presented for the effects of favorable streamwise mean pressure gradients on temporal entropy generation rates and, in the process, on key Reynolds-stress-producing events such as sweep front passage and on the deceleration/outflow phase of the overall bursting process. Two extremes have been considered: (1) a high pressure gradient, nearing "laminarization," and (2) for comparison, a low pressure gradient corresponding to many earlier experiments. In both cases, the peak temporal entropy generation rate occurs shortly after passage of the ejection/sweep interface. Whether sweep and ejection rates appear to decrease or increase with the pressure gradient depends on the feature examined and the manner of sampling. When compared using wall coordinates for velocities, distances and time, the trends and magnitudes of the transient behaviors are mostly the same. The main effects of the higher pressure gradient are (1) changes in the time lag between detections - representing modification of the shape of the sweep front and the sweep angle with the wall, (2) modification of the magnitude of an instantaneous Reynolds shear stress with wall distance and (3) enlarging the sweeps and ejections. Results new for both low and high pressure gradients are the temporal behaviors of the dominant contribution to entropy generation; it is found to be much more sensitive to distance from the wall than to streamwise mean pressure gradient.
\end{abstract}




\section{ACKNOWLEDGEMENTS}

We appreciate the kindly assistance of Prof. James M. Wallace of U. of Maryland, an architect of the original pattern recognition code, and Prof. Ronald L. Panton of U. Texas who also provided advance copies of their recent journal manuscripts. The study reported was partly supported through the Long Term Research Initiative at the Idaho National Engineering Laboratory under DoE Idaho Operations Office Contract DE-AC07-76ID01570. Earlier incarnations were financed by the Applied Hydrodynamics Research Program of the Office of Naval Research (Dr. James A. Fein, Program Manager), the National Science Foundation (Dr. Win Aung, Program Manager), the U.S.-Deutschland Fulbright Commission, the Max Planck Gesellschaft, the University of Arizona and Westinghouse Naval Systems Division (earlier Gould Ocean Systems Division). At the University of Limerick, Prof. Mark R. D. Davies and Dr. Edmond J. Walsh provided friendly encouragement and support enabling completion of this report. To all we are extremely grateful. 


\section{CONTENTS}

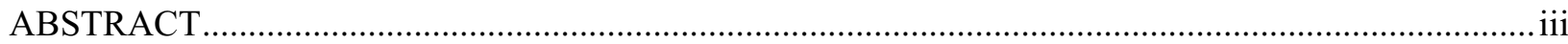

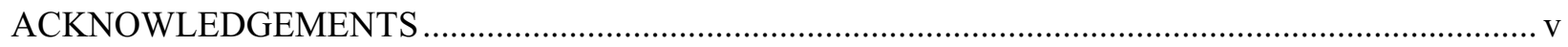

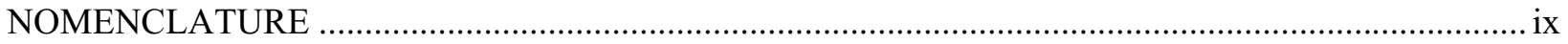

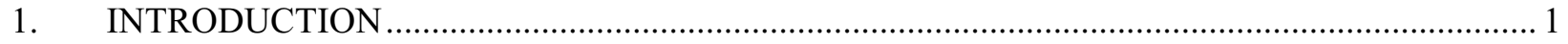

1.1 Background on Entropy Generation............................................................................... 2

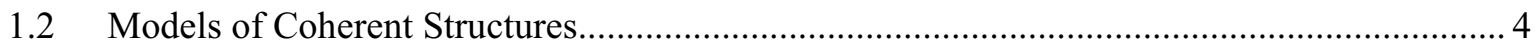

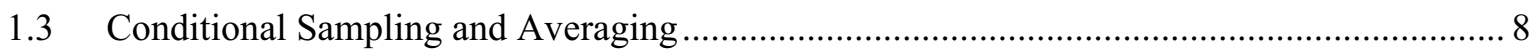

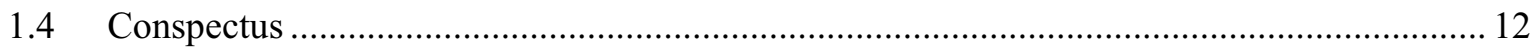

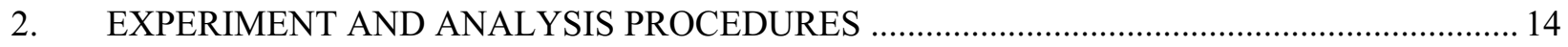

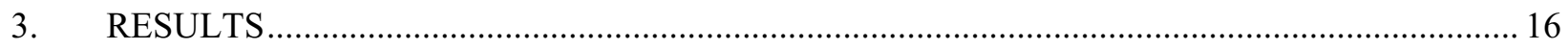

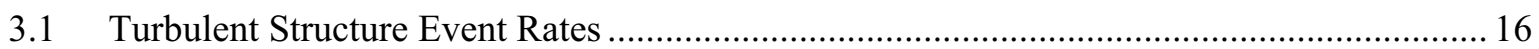

3.1.1 Reference Condition - Low Pressure Gradient ........................................................ 16

3.1.2 Effects of Pressure Gradient .............................................................................. 19

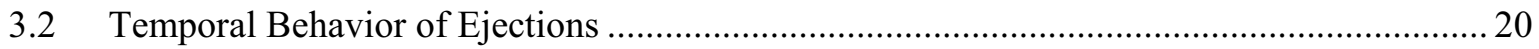

3.3 Temporal Behavior of Sweeps as Identified via Pattern Recognition................................. 21

3.4 Effects of Pressure Gradient on Temporal Behavior of Sweeps …...................................... 22

3.5 Temporal Behavior of Entropy Generation Rates and Effects of Pressure Gradient ............ 27

3.6 Implications for Turbulence Modeling …...................................................................... 31

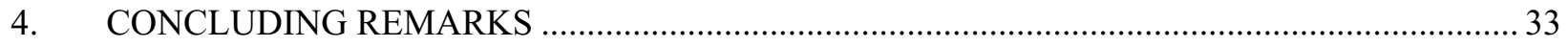

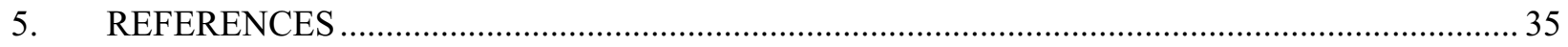




\section{FIGURES}

Figure 1. Sweeps and ejections identified across the viscous layer non-dimensionalized with wall coordinates from conditional sampling by the Vita technique (sweeps, circles) [Blackwelder and Kaplan, 1976], by pattern recognition, called PatRec (sweeps, squares) [Wallace, Brodkey and Eckelmann, 1977] and by quadrant splitting (triangles) [Wallace, Eckelmann and Brodkey, 1972]: (a) totals identified and (b) non-dimensional rates. Symbol Q2 represents ejections and Q4 sweeps; solid lines connect data at the higher streamwise pressure gradient $\left(-\mathrm{K}_{\mathrm{p}} \approx 0.02\right)$ and dashed ones are for the lower pressure gradient $\left(-\mathrm{K}_{\mathrm{p}} \approx 0.008\right)$.

Figure 2. Transient behavior of abrupt ejections identified by the quadrant splitting technique: (a) lower pressure gradient $\left(-\mathrm{K}_{\mathrm{p}} \approx 0.008, \mathrm{y}^{+} \approx 13.2\right)$ and $(\mathrm{b})$ higher pressure gradient $\left(-\mathrm{K}_{\mathrm{p}}\right.$ $\approx 0.02, \mathrm{y}^{+} \approx 16$ )

Figure 3. Comparison of transient behaviors of sweeps identified by pattern recognition technique (dashed curves) and Vita technique (solid curves) at $\mathrm{y}^{+} \approx 13.2$ in viscous layer of the lower pressure gradient experiment.

Figure 4. Transient behavior of sweeps for hpg (top) and lpg (bottom) at three comparable locations across the viscous layer. The non-dimensional time $\mathrm{t}_{\mathrm{W}}{ }^{+}=0$ is based on equivalent Vita detection for the $\tau_{\mathrm{W}}\{\mathrm{t}\}$ signal. (---------, $<\mathrm{u}>^{+}$; ---- ----, $<_{\mathrm{V}}>^{+}$; -- -,$\left.--<\mathrm{uv}>^{+} ;----\cdot \cdot---,<\tau_{\mathrm{W}}{ }^{+}\right)$

Figure 5. Front shape as indicated by non-dimensional time lag between $\mathrm{u}$-detection and $\tau_{\mathrm{W}^{-}}$ detection (circles, $-\mathrm{K}_{\mathrm{p}} \approx 0.02$; squares, $-\mathrm{K}_{\mathrm{p}} \approx 0.008$ ).

Figure 6. Transient behavior of the dominant contribution to pointwise total entropy generation rate; high pressure gradient case $\left(-\mathrm{K}_{\mathrm{p}} \approx 0.02\right)$ identified with solid curves and low pressure gradient $\left(-\mathrm{K}_{\mathrm{p}} \approx 0.008\right)$ with dashed curves: (a) at probe locations for lpg experiment and (b) for hpg experiment.

\section{TABLES}

Table 1. Non-dimensional times of occurrences of interesting events in the Vita signatures (all times in terms of $\mathrm{t}_{\mathrm{W}}{ }^{+} ; \mathrm{t}_{\mathrm{W}}{ }^{+}=0$ at equivalent wall detection). 


\section{NOMENCLATURE}

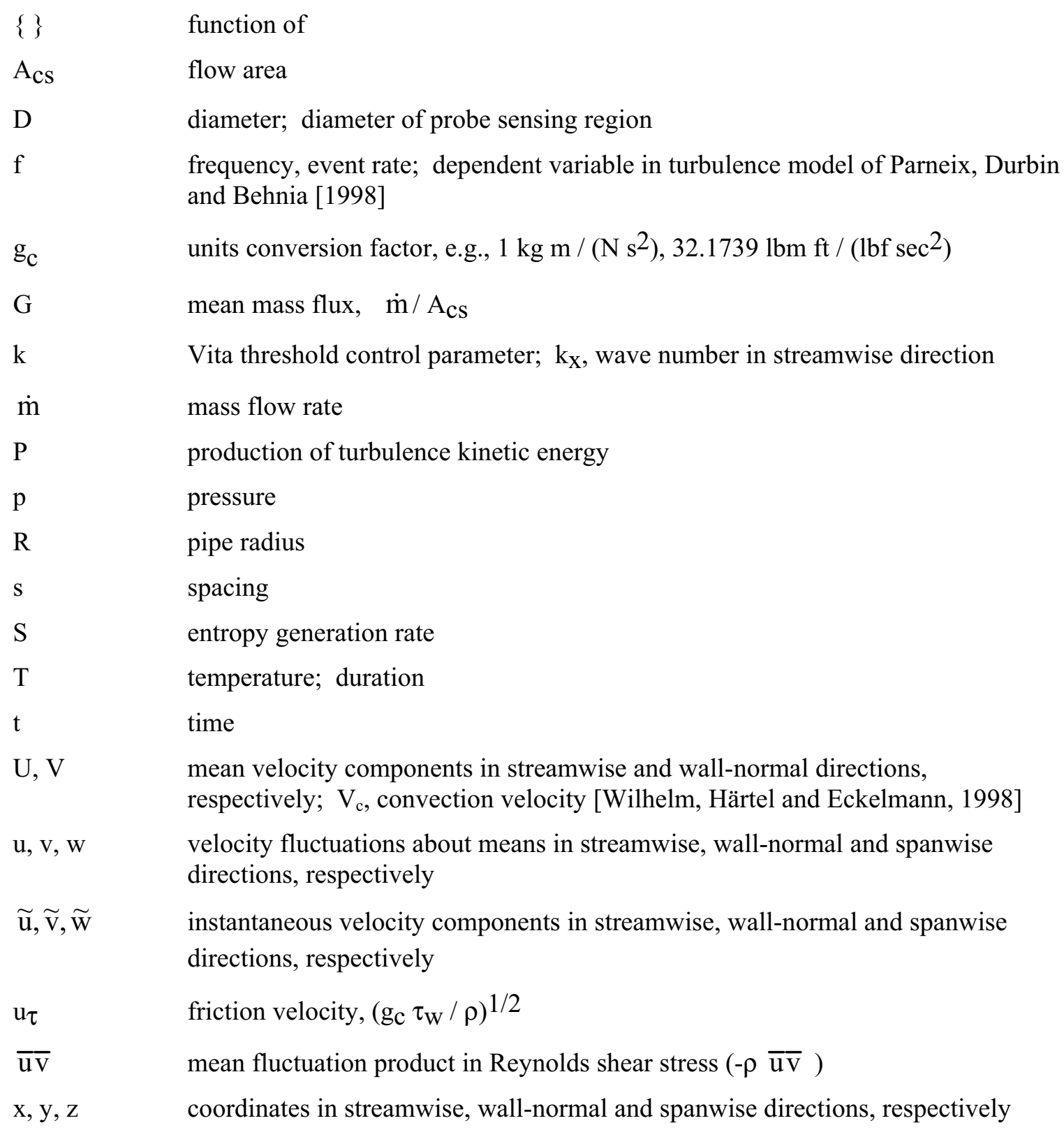

$\underline{\text { Non-dimensional quantities }}$

$\begin{array}{ll}\mathrm{cf}_{\mathrm{f}} & \text { skin friction coefficient, } 2 \mathrm{gc}_{\mathrm{c}} \tau_{\mathrm{W}} /\left(\rho \mathrm{U}_{\infty}{ }^{2}\right) \\ \mathrm{f}^{+} & \text {event rate, } \mathrm{fv} / \mathrm{u} \tau^{2} \\ \mathrm{~K}_{\mathrm{p}} & \text { streamwise pressure gradient, }\left(\mathrm{v} / \mathrm{\rho u}_{\tau}{ }^{3}\right) \mathrm{dp} / \mathrm{dx} \\ \mathrm{K}_{\mathrm{V}} & \text { acceleration parameter, }\left(\mathrm{v} / \mathrm{U}_{\infty}{ }^{2}\right) \mathrm{dU} \mathrm{U}_{\infty} / \mathrm{dx}\end{array}$




$\begin{array}{ll}\mathrm{Re} & \text { Reynolds number; ReD, based on diameter, } \mathrm{G} \mathrm{D} / \mu \\ \mathrm{Re}_{\tau} & \text { distance from wall to centerplane, centerline, etc., } \mathrm{y}_{\mathrm{c}} \mathrm{u}_{\tau} / \nu \\ \mathrm{S}^{+}, \mathrm{S}_{\mathrm{t}}^{+} & \text {pointwise volumetric entropy generation rate, } \mathrm{T} v \mathrm{~S}^{\prime \prime \prime} /\left(\rho \mathrm{\rho} \tau^{4}\right) \\ \mathrm{t}^{+} & \text {time, } \mathrm{t} \mathrm{u} \tau^{2 / \nu} \\ \mathrm{y}^{+} & \text {wall-normal coordinate, } \mathrm{y} \mathrm{u} \tau / \nu \\ \delta^{+} & \text {boundary layer thickness, } \delta \mathrm{u} \tau / \nu \\ \varepsilon^{+} & \text {turbulent dissipation of turbulent kinetic energy, } v \varepsilon / \mathrm{u} \tau \\ \tau^{+} & \text {pointwise shear stress, } \tau\left\{\mathrm{y}^{+}\right\} / \tau_{\mathrm{W}}\end{array}$

\section{$\underline{\text { Greek symbols }}$}

$\delta \quad$ boundary layer thickness

$\varepsilon \quad$ dissipation of turbulence kinetic energy

$\mu \quad$ absolute viscosity

$v \quad$ kinematic viscosity, $\mu / \rho$

$\rho \quad$ density

$\tau \quad$ shear stress; $\tau_{\mathrm{W}}$, wall shear stress

$\Phi \quad$ viscous dissipation function

$\omega \quad$ vorticity fluctuation

\section{$\underline{\text { Superscripts }}$}

()$^{+} \quad$ normalization by wall units, $v$ and $\mathrm{u} \tau$

(_) root-mean-square value

()$^{\prime \prime \prime} \quad$ per unit volume

( ) time mean value

(_) instantaneous value

$<>\quad$ ensemble average 


\section{$\underline{\text { Subscripts }}$}

$\begin{array}{ll}\text { avg } & \text { average } \\ \mathrm{b} & \text { bulk or mixed-mean quantity (one-dimensional); burstung } \\ \mathrm{c} & \text { centerplane, centerline } \\ \mathrm{cs} & \text { cross section } \\ \mathrm{D} & \text { evaluated with diameter } \\ \max & \text { maximum } \\ \min & \text { minimum } \\ \mathrm{t} & \text { total } \\ \mathrm{u} & \text { relative to u detection } \\ \mathrm{uy} & \text { partial derivative of u with respect to y } \\ \text { W } & \text { wall, wallward, relative to wall detection } \\ \infty & \text { freestream value }\end{array}$




\title{
Temporal Entropy Generation in the Viscous Layers of Laterally-Converging Duct Flows
}

\author{
Donald M. McEligot ${ }^{1,2,3,4}$, Robert S. Brodkey ${ }^{5}$ and Helmut Eckelmann ${ }^{6,7}$
}

1. Aero. Mech. Engr. Dept., Univ. Arizona, Tucson, Ariz. 85721 USA

2. Inst. für Kernenergetik und Energiesysteme (IKE), Uni. Stuttgart, D-70569 Stuttgart, Deutschland

3. Idaho National Laboratory (INL), Idaho Falls, Ida. 83415-3885 USA

4. Stokes Research Institute, Univ. Limerick, Limerick, Ireland

5. Chem. Engr. Dept., Ohio State Univ., Columbus, Ohio 43210 USA

6. Institut für Nichtlineare Dynamik, Universität Göttingen, D37073 Göttingen, Deutschland

7. Max-Planck-Institut für Dynamik und Selbstorganisation (formerly Max-Planck-Institut für Strömungsforschung), Bunsenstr. 10, D37073 Göttingen, Deutschland.

\section{INTRODUCTION}

For wall-bounded flows most entropy is generated in the viscous layer and knowledge of the entropy generation process is a key to reducing fuel consumption and/or waste by increasing efficiency, so we study the transient behavior of its dominant contributor there for a favorable streamwise gradient, a non-canonical flow warranting more research. Our approach is to extend the time series data of McEligot and Eckelmann [2006] to provide simultaneous ensemble-averaged distributions of this contribution in the vicinity of inclined shear layer (or sweep front) passage when it is maximal. In the process, new fundamental knowledge is obtained concerning the effects of pressure gradients on the turbulent structures known as sweeps and ejections and on their event rates.

As pointed out by McEligot and Eckelmann [2006], laterally converging flow is one version of a group of flows which lead to favorable streamwise pressure gradients and, consequently, may modify characteristics of a turbulent flow. "Strong" favorable pressure gradients have been found to reduce drag by altering the structure of the turbulent velocity fluctuations. In fact, with a favorable non-dimensional pressure gradient $\mathrm{K}_{\mathrm{p}}=\left(\mathrm{v} / \mathrm{\rho u}_{\tau}{ }^{3}\right) \mathrm{dp} / \mathrm{dx}$ on the order of -0.03 an apparent "laminarization" of a turbulent boundary layer (TBL) can occur [Narasimha and Sreenivasan, 1979]. (Here $v, \rho, u \tau, p$ and $x$ denote kinematic viscosity, fluid density, friction velocity, static pressure and streamwise coordinate, respectively.) Others include sink flows [Spalart, 1986; Haas and Schneider, 1996; Jones, Marusic and Perry, 2001], flows around the leading edges of wings and turbine blades and vanes [Mayle, 1991], rocket nozzles [Launder, 1964] and fully-developed duct and tube flows [Kim, Moin and Moser, 1987; Durst, Jovanovic and Sender, 1995]. Related previous studies have been summarized by Narasimha and Sreenivasan [1979], Murphy, Chambers and McEligot [1983], Spalart [1986] and McEligot and Eckelmann (hereinafter called ME). As noted by Johansson, Alfredsson and Kim in 1991, little is known about turbulence in non-canonical situations such as significant pressure variation; this comment appears to be valid still.

This study is the fourth in a sequence delving successively deeper into the structure of laterally converging turbulent flows. The general question being addressed is - how is the turbulence structure in the viscous layer modified by the favorable mean streamwise pressure gradient induced by such a convergence? The first paper considered the mean flow in terms of the streamwise pressure distribution and wall shear stress as well as applying a simple mixing length model for their numerical prediction; we 
will refer to this paper as MCM [Murphy, Chambers and McEligot, 1983]. The second by Chambers, Murphy and McEligot [1983], say CMM, measured the temporal wall shear stress and analyzed it via the Vita technique of Blackwelder and Kaplan [1976]. The experiment of ME and the present study concentrate on the viscous layer (defined as the region where viscous effects are significant, but not necessarily dominant [Bradshaw, 1975], typically taken to a distance from the wall $\mathrm{y}^{+}=\mathrm{y}_{\tau} / \mathrm{v}$ of about thirty in a classical zero-pressure gradient case - it includes the "laminar" and "buffer" sublayers in some investigators' terminology). The superscript " + " represents non-dimensionalization by $v$ and the friction velocity $\mathrm{u}_{\tau}=\left(\tau_{\mathrm{W}} / \rho\right)^{1 / 2}$ with $\tau_{\mathrm{W}}$ symbolizing the mean wall shear stress. This viscous layer typically is the region where the largest gradients occur and the turbulence production is largest. The major resistances to momentum, energy and mass transfer occur in this layer - and the pointwise entropy generation rate per unit volume $S^{\prime \prime \prime}$ is greatest here as well. While some investigators may consider the viscous layer to extend to $\mathrm{y}^{+} \approx 100$, the direct entropy generation rate decreases to less than one per cent of its wall value by $\mathrm{y}^{+} \approx 30$.

Based on the results of ME and the earlier studies cited by them, we can summarize the key observations concerning modifications of the mean turbulence structure in the viscous layer by favorable streamwise pressure gradients. There is evidence that the turbulence structure is modified. So it has been important (1) to determine which features remain relatively invariant in the viscous layer and (2) to quantify the variation of the others in order to provide the turbulence structure information needed in models accounting for their effects. An effect of a favorable pressure gradient is to "thicken" the viscous layer, in some senses; bursting rates are apparently reduced [Kline et al., 1967; CMM; Finnicum and Hanratty, 1988]. The consequent upward shift in the logarithmic region as the Reynolds number is lowered was identified earlier via the mean velocity measurements of Senecal [1952; McEligot, Ormand and Perkins, 1966]. Throughout the remainder of this article, the streamwise velocity is represented as

$\tilde{\mathrm{u}}=\mathrm{U}+\mathrm{u}$, where the tilde denotes an instantaneous quantity and upper and lower case letters symbolize the corresponding mean value and the fluctuation about it, respectively; the normal velocity $\tilde{\mathrm{V}}$ is treated in a similar fashion.

\subsection{Background on Entropy Generation}

Understanding of the behavior of the entropy generation rate is important for several reasons. The local (pointwise) entropy generation rate per unit volume $\mathrm{S}^{\prime \prime}$ is a key to improving many energy processes and applications [Bejan, 1982]. Bejan has suggested that real systems which owe their thermodynamic imperfections to fluid flow, heat transfer and mass transfer irreversibilities be optimized by minimizing their entropy generation. This approach has been applied to compact heat exchangers, power plants, natural convection, rotating bodies, enhanced heat transfer surfaces, impinging jets, convection in general and other thermal systems. Since the loss of available work [Kestin, 1980] is proportional to the amount of entropy produced (e.g., Btu/R or J/K), apparatus producing less entropy by irreversibilities destroys less available work, increasing the efficiency and, in turn, reducing fuel consumption and waste products. The pointwise S"' determines the localized contribution to energy losses or reduction in the availability of energy [Clausius, 1887], so insight into the dominant loss sources and their locations can allow reducing them intelligently, thereby improving efficiency. Kock and Herwig [2004], Neumann, von Wolfersdorf and Weigand [2005] and others are using computational fluid dynamics (CFD) codes to predict the distribution of entropy generation for optimization by minimizing it.

Previous predictions of the time-mean behavior in turbulent channel flows and boundary layers have shown that $\left(\mathrm{S}^{\prime \prime \prime}\right)^{+}$increases as the wall is approached, that most entropy generation occurs in our viscous layer, that there is no significant dependence on Reynolds number in flows with negligible $\mathrm{dp} / \mathrm{dx}$ and that there can be expected to be slight effects of favorable $K_{p}$ [Bejan, 1982; McEligot et al., 2008a,b]. So we 
know in general where significant time-mean entropy generation occurs; in a sense, our present interest is to find when it occurs in terms of apparent coherent structures. Therefore we deduced measurements of temporal entropy generation which are new for both lpg (low pressure gradient) and hpg (high pressure gradient) conditions.

As shown by Bejan [1982] and others, the entropy generation rate per unit volume is given by $\mathrm{S}^{\prime \prime \prime}=\mu \Phi / \mathrm{T}$, where $\Phi$ is the dissipation function and $\mathrm{T}$ is the absolute temperature. This observation is one reason that dissipation of kinetic energy may be of interest to some. The instantaneous dissipation function is given by Schlichting [1982, eq. 12,8] and others. In wall coordinates, one may evaluate the instantaneous entropy generation rate for incompressible flows as

$$
\begin{gathered}
\mathrm{S}_{\mathrm{t}}^{+}=2\left[\left(\tilde{\partial \mathrm{u}}+/ \partial \mathrm{x}^{+}\right)^{2}+\left(\partial \tilde{\mathrm{v}}^{+} / \partial \mathrm{y}^{+}\right)^{2}+\left(\tilde{\mathrm{w}}+/ \partial \mathrm{z}^{+}\right)^{2}\right]+\left[\left(\partial \tilde{\mathrm{v}}^{+} / \partial \mathrm{x}^{+}\right)+\left(\partial \tilde{\mathrm{u}}+/ \partial \mathrm{y}^{+}\right)\right]^{2} \\
+\left[\left(\partial \tilde{\mathrm{w}}^{+} / \partial \mathrm{y}^{+}\right)+\left(\partial \tilde{\mathrm{v}}+/ \partial \mathrm{z}^{+}\right)\right]^{2}+\left[\left(\partial \tilde{\mathrm{u}}^{+} / \partial \mathrm{z}^{+}\right)+\left(\partial \tilde{\mathrm{w}}^{+} / \partial \mathrm{x}^{+}\right)\right]^{2}
\end{gathered}
$$

where $\mathrm{S}_{\mathrm{t}}{ }^{+}$is defined as $\mathrm{T} v \widetilde{\mathrm{S}}$ "'/( $\left.\rho \tau_{\tau}^{4}\right)$ with the tilde again denoting the instantaneous total value. Integration of this function with respect to time gives the familiar relations for dissipation of kinetic energy of the mean motion plus dissipation of turbulence kinetic energy $\varepsilon$, e.g., as by Rotta [1962, eq. 7.1] under boundary layer approximations and by Gersten and Herwig [1992, eqs. A1.21 and A1.25] for more general flows. These quantities are called "direct" (or mean) and "indirect" (or turbulent) dissipation, respectively, by authors studying entropy generation [Kock and Herwig, 2004]. One might question the physical meaning of splitting entropy generation into two parts (direct and turbulent). Since - in reality the mean profile never occurs, the direct part might only be described as "something that would exist if the flow were steady and laminar with a profile like the turbulent mean one." The same question applies to Reynolds averaging in general. Comparable concerns have been presented by Brodkey et al. [1973, 1974] and Bradshaw [1974] concerning the idea of "instantaneous production" of turbulent kinetic energy (tke). However, in the present study, we aim to examine the transient behavior of the instantaneous entropy generation (as defined above) and, therefore, the separation into "mean" and "turbulent" is not involved. In unheated flows with entropy generation solely due to friction as here, one can see that the instantaneous entropy generation rate is the same as the "instantaneous total dissipation rate." However, thinking of entropy generation seems more appropriate for considering efficiency, lost work, availability, etc. - and it extends directly to cases with additional irreversibilities, such as significant heat transfer [Kock and Herwig, 2004].

Some further insight does come from studies concentrating on the time-mean dissipation of turbulent kinetic energy. From analysis and application of boundary layer assumptions for asymptotic high-Reynolds-number zero-pressure-gradient (zpg) and of measurements by Klebanoff [1955] at $\delta^{+} \approx 2200$, Rotta [1962] has concluded that almost sixty per cent of the dissipation (and therefore entropy generation) occurs within $\mathrm{y}^{+}$less than about twenty. The rate of direct dissipation alone amounts to forty per cent of the total energy loss and nearly twenty per cent is converted into turbulent energy in the same region. With their 2-1/2 D model for the viscous layer Finnicum and Hanratty [1988] have forecast that, relative to a zpg boundary layer, a near-laminarizing pressure gradient will yield an increase in direct dissipation and will lower indirect dissipation but they did not present the effects on their sum (i.e., $\mathrm{St}^{+}$). Antonia, Kim and Browne [1991] examined turbulent dissipation $\varepsilon^{+}\left(=v \varepsilon / u \tau^{4}\right)$ and models thereof for turbulent channel flows at $\operatorname{Re}_{\tau}=\left(\mathrm{su}_{\tau} /(2 \mathrm{v})\right)=180$ and $395\left(-\mathrm{K}_{\mathrm{p}} \approx 0.0056\right.$ and 0.0025 , respectively) as predicted by the DNS (direct numerical simulation) algorithm of Kim, Moin and Moser [1987]. The quantity $\mathrm{s}$ is the spacing between the channel walls. This Reynolds number is also the value of $\mathrm{y}^{+}$at the 
centerplane $\left(\mathrm{yc}_{\mathrm{c}}^{+}\right)$. Their results show that, for this limited range, $\varepsilon^{+}$decreased as $\left|-\mathrm{K}_{\mathrm{p}}\right|$ increased, consistent with the observations of Spalart [1986] for boundary layers. Indirectly, in a study of anisotropy of turbulence Jovanovic and Hillerbrand [2005] have demonstrated that the turbulent dissipation rate at the wall decreases as a favorable pressure gradient increases. This trend corresponds to an approach to their "one-component limit" and accompanying suppression of small-scale turbulence in near-wall layers. Laadhari [2007] examined the effects of Reynolds number in channel flows on an overall dissipation function (evaluated via a "production function"), emphasizing the asymptotic high-Reynolds-number limit.

In boundary layers or comparable channel flows the term $\left(\partial \mathrm{U}^{+} / \partial \mathrm{y}^{+}\right)^{2}$ dominates the direct dissipation as noted by Rotta. Likewise, Antonia, Kim and Browne [1991] indicate that in the viscous layer the term $\left(\partial \mathrm{u}^{+} / \partial \mathrm{y}^{+}\right)^{2}$ provides the dominant contribution to the indirect (turbulent) dissipation.

Consequently, $\left(\tilde{\mathrm{u}}^{+} / \partial \mathrm{y}^{+}\right)^{2}$ can be expected to be most important in evaluating $\mathrm{S}_{\mathrm{t}}{ }^{+}$. In studying the passage of internal turbulent shear layer structures, Johansson, Alfredsson and Eckelmann [1987] and Johansson, Alfredsson and Kim [1991] demonstrated that $\partial \mathrm{u} / \partial \mathrm{y}$ is greatest and considerably greater than $\partial \mathrm{u} / \partial \mathrm{x}$ there. Thus, it is $\partial \mathrm{u} / \partial \mathrm{y}$ that is expected to be a dominant contributor to temporal entropy generation. It will later be shown that the maxima of $\partial \mathrm{u} / \partial \mathrm{y}$ can be identified by detection of the maxima of $\partial \mathrm{u} / \partial \mathrm{t}$ in the times series data.

The mean turbulence structure of the viscous layer with favorable pressure gradients is reasonably well understood now. The present objectives then are to determine whether streamwise mean pressure gradients affect the temporal behavior of entropy generation rates in the viscous layer and, if so, which aspects are affected significantly. The method of determination is by analysis of the time series measurements of ME. These data provide means to deduce the transient variation of the dominant contribution $\mathrm{S}_{\mathrm{t}, \mathrm{uy}}{ }^{+}=\left(\tilde{\partial \mathrm{u}^{+}} / \partial \mathrm{y}^{+}\right)^{2}$ and, in the process, yield information on effects of pressure gradient on structure event rates and ensemble-averaged $\left\langle\mathrm{u}^{+}\left\{\mathrm{t}^{+}\right\}>,<\mathrm{v}^{+}\left\{\mathrm{t}^{+}\right\}>\right.$and $<\mathrm{uv}\left\{\mathrm{t}^{+}\right\}>^{+}$. These latter time series give some guidance on the effects of pressure gradient on hypothesized structures. (The brackets indicate an ensemble average and the additional subscripts uy denote the partial differentiation of $u$ with respect to y for $\mathrm{S}_{\mathrm{t}, \mathrm{uy}}{ }^{+}$.)

\subsection{Models of Coherent Structures}

Over the last several decades, many models have been hypothesized to explain the behavior of fluid parcels in the important viscous layer and beyond [Theodorsen, 1952; Townsend, 1956, 1961; Kline et al., 1967; Corino and Brodkey, 1969; Bejan, 1982; Robinson, 1991; Morrison, Subramanian and Bradshaw, 1992; Morrison and Westbury, 1996; Panton, 1997, 2001, 2005; Adrian, Meinhart and Tomkins, 2000; Tomkins and Adrian, 2003; Adrian, 2007; Morrison 2007; and others]. These structures have been described variously as spaghetti, hairpins, bursts, ejections, inverted mushrooms, splats, quasi-streamwise vortices, sweeps, horseshoes, attached eddies, buckling layers, legs, necks, splashing, arches, rolls, low-speed streaks, pockets, turbulent bulges, ultimate eddies and typical eddies.

So called sweeps or fronts, which are events in a turbulent flow that displace the surrounding media due to their higher velocity, were first described by Corino and Brodkey [1969] in their visual studies and were further described by Nychas, Hershey and Brodkey [1973]. An ejection is an abrupt movement outward from the wall area of fluid originally within this region. It occurred immediately after the start of an acceleration process and originated within the mass of fluid that constituted the prior retarded element. Often there was associated with these events a zone of high shear at the interface between the mean flow and the decelerated region that gave rise to the ejected element. Most ejections originated in the regions 
from $\mathrm{y}^{+}$about five to fifteen. Brodkey et al. [1974] note that total dissipation (and therefore instantaneous total entropy generation rate) can be considered as a composite of local processes, one of which (observed visually) occurs in the high velocity gradient region that separates the decelerated flow (from which ejections originate) from the higher-speed sweep.

From the visual studies [Corino and Brodkey, 1969; Nychas, Hershey and Brodkey, 1973], one may derive a conceptual physical model of the motions in the burst sequence. When one observes sweeps, one sees a high speed parcel (relative to the local mean velocity) approaching the wall at a shallow angle. This high speed fluid pushes slower fluid in front of it onward; we picture this process as ejections of low speed fluid away from the wall. The causative factor is the high speed fluid inward and the result is low speed fluid moving outward. The interaction terms (always somewhat less important) are consequences when the locally-averaged velocities are either above or below the mean velocity. While other secondary motions occur in this three-dimensional flow, these phenomena are the main ones represented by the ensemble averages of sensor response at a point. What does this simplistic picture imply? The sweeps coming from the outer region are relatively large and progress across the entire viscous layer until impeded and slowed by the wall; thus, the rate of sweeps should be relatively constant across the layer. Very close to the wall, the events are constricted to the wall direction, i.e., parallel to the wall. It would be logical that, very close to the wall, one would see a reduction in rate of ejections. But, over the rest of the layer, the induced ejection rate should be constant since they are part of the same event sequence no matter where they are deduced.

Wallace, Eckelmann and Brodkey [1972] "associated" sweeps with events having positive u- and negative v-fluctuations (or "quadrant 4" ----> Q4) relative to the time-mean velocity in an Eulerian coordinate system. However, this definition does not exactly describe the essential characteristic of sweeps as being fronts of accelerated fluid. As known from the visual studies, these fronts are associated with large positive instantaneous gradients $\tilde{\partial \mathrm{u}} / \partial \mathrm{t}$ and with large positive spatial gradients $\partial \tilde{\mathrm{u}} / \partial \mathrm{y}$. Kreplin and Eckelmann [1979b] observed that a steep front occurs in the near-wall region, out to $\mathrm{y}^{+}$about fifty, with a convection velocity varying with wall distance. Due to the steep slope of this front, disturbances further from the wall are observed later at locations nearer the wall than away from the wall. Brodkey, Wallace and Eckelmann [1974] were careful to point out that there does not exist a one-to-one correspondence between the visually-observed motions and the quadrant categorizations. In particular, not all Q2 contributions correspond to the small, abrupt ejections defined by Corino and Brodkey. In the present paper the term burst will refer to the overall process (and not just the ejections). Since not all Q2 events represent the abrupt small ejections emphasized by Corino and Brodkey (although they are included) and not all Q4 events represent sweep front passage, we will attempt to discriminate between these differences. The words "ejection" and "sweep" will be applied to indicate those motions as described by Corino and Brodkey. When simply classifying any motion with an outward v-fluctuation and decelerating u-fluctuation, the term "Q2 event" should be used. Likewise sweeps and sweep fronts should be discriminated from "Q4 events" overall. While sweeps/fronts could be characteristics of a number of the proposed descriptions, such as splats, inverted mushrooms, wobbling streamwise vortices and "typical" eddies, one would not expect to be able to discriminate which, if any, of these proposed models are valid via one-dimensional temporal measurements like the present database.

Johansson, Alfredsson and Eckelmann [1987] used the Vita technique and simultaneous measurements from two sensors to construct the temporal and, hence, spatial fields in the vicinity of internal shear layer passage. Their constructed spatial field shows maximum values of $\partial u / \partial x$ and $\partial u / \partial y$ at detection (passage). By applying their Visa technique, which detects the passage of inclined internal shear layer structures, Johansson, Alfredsson and Kim [1991] were able to construct comparable spatial and temporal behavior of $<\mathrm{u}>,<\mathrm{v}>,<\mathrm{uv}>,<\mathrm{p}>$ and turbulence production in the vicinity of this event from DNS of channel flow with a slight to moderate streamwise pressure gradient. Their 
conditionally-averaged spatial fields in the x-y plane provide useful insight for interpreting the present Vita deductions for lpg. While Visa essentially triggers on high values of instantaneous $\partial \mathrm{u} / \partial \mathrm{x}$ (analogous to the $\partial \mathrm{u} / \partial \mathrm{t}$ of Vita), their Figures 4 and 5 demonstrate that $\partial \mathrm{u} / \partial \mathrm{y}$ is considerably greater than $\partial \mathrm{u} / \partial \mathrm{x}$ there. It is $\partial \mathrm{u} / \partial \mathrm{y}$ that is expected to be a dominant contributor to temporal entropy generation.

With DNS Wilhelm, Härtel and Eckelmann [1998], say WHE, found further evidence that the fronts first discussed by Kreplin and Eckelmann [1979b] are essentially identical to extended shear layers in near-wall flow. In good agreement with the experimental results, the front was found to be inclined to the wall from the linear layer into the logarithmic region. (The linear layer is the region where the flow is dominated by molecular transport, typically to $\mathrm{y}^{+}$about five and often called the laminar sublayer.) The deductions of temporal turbulence production rates $<\mathrm{P}>$ show they can significantly exceed the values deduced from $<$ uv $>$ dU/dy commonly used [Diorio, Kelley and Wallace, 2007] and that the differences are greatest in the region of the inclined shear layer. Wilhelm, Härtel and Eckelmann also note that turbulent dissipation was found to be quite large in the vicinity of the shear layer. The spatial distributions show persistent, long motion of low-speed fluid away from the wall (Q2) downstream of the detection (corresponding to the time earlier than a Vita detection) as suggested in the visual studies of Corino and Brodkey [1969]. Consequently, they conclude that the dominant contribution to $<u v>$ is from ejection type motions downstream of their detection.

Several recent studies have proposed ideas, models, coherent structures and mechanisms to explain why wall turbulence is "self-sustaining" [Robinson, 1991; Panton, 1997, 2001; Bernard and Wallace, 2002; Adrian, 2007]. These studies typically have concentrated on fully-developed pipe or "infinitely-wide" channels or on zero-pressure gradient boundary layers; consequently, effects of favorable pressure gradients have generally not been considered (even when significant as in low-Reynolds-number channel flows). A simple-minded view is that, in fully-developed duct flows, wall turbulence is sustained by the continual pressure loss and, in boundary layers, by the continual transfer of momentum from the freestream. For the fully-developed duct flow, the consequent skin friction coefficient is a function of a single parameter, the Reynolds number, so all the various motions - that have been observed or hypothesized - must organize themselves so that this function is satisfied. (A corollary of this situation is that the fewer constants/functions in a turbulence model, the easier it should be to satisfy this requirement.) As noted by Johansson, Alfredsson and Kim [1991], little is known about turbulence in non-canonical situations such as significant pressure variation; this comment appears to be valid still.

A potential benefit of the detailed structure studies could be to identify their dominant aspects and how they would be modified by pressure gradients, property variation, compressibility or other phenomena and thereby be able to predict the effects of these phenomena. For example, the mean total shear stress profile, which the hypothesized motions must provide, may be approximated [Rotta, 1962; Finnicum and Hanratty, 1988] as

$$
\tau^{+}\left\{\mathrm{y}^{+}\right\}=\left(\tau\left\{\mathrm{y}^{+}\right\} / \tau_{\mathrm{w}}\right)=1+\mathrm{K}_{\mathrm{py}^{+}}-\mathrm{K}_{\mathrm{py}} \mathrm{y}^{+}\left(\mathrm{c}_{\mathrm{f}} /\left(2 \mathrm{y}^{+}\right)\right) \int_{0}^{\mathrm{y}^{+}}\left(\mathrm{U}^{+}\right)^{2} \mathrm{dy}^{+}
$$

near the surface. The skin friction coefficient $\mathrm{c}_{\mathrm{f}}$ is $2 \tau_{\mathrm{W}} /\left(\rho_{\mathrm{b}}{ }^{2}\right)$ where $\mathrm{Ub}_{\mathrm{b}}$ is a characteristic fluid velocity. For a flow with zero pressure gradient it is inherently unity while, for fully-developed channel flow, the second term on the right becomes significant at low Reynolds numbers (as seen by the identity $-K_{p}=1 / \operatorname{Re}_{\tau}$ ) and could be equivalently called a low Reynolds number effect. For the converging flow the advection term is slight but observable in our viscous layer; McEligot and Eckelmann [2003] demonstrated a slight effect on deduced $\mathrm{u}_{\tau}$ for $-\mathrm{K}_{\mathrm{p}} \approx 0.01$ at $\mathrm{y}^{+} \approx 25$ near its outer edge. The advection 
term becomes more important beyond the viscous layer where some of the hypothesized motions are suggested to originate.

As sketched by Andreopoulos and Agui [1996] (their Fig 30a) a fluid parcel impinging obliquely with a surface can be expected to induce streamwise vortices. This idea was also discussed by McEligot in the context of "bypassed" transition [APS Bull. Sheet, 2006]. Suppression of fluctuating wallward motions is likely a key to the process which yields universal behavior near the wall for simple canonical flows and a skin friction correlation with a single independent variable (the latter may also be deduced with appropriate assumptions and dimensional analysis).

Panton [1997, 2001] provides an excellent review of the models which have been hypothesized to explain self-sustaining wall turbulence in zpg boundary layers and fully-developed duct flows. He explains that there are two main categories: (1) parent vortices interact with the wall and produce offspring vortices and (2) instabilities evolving in long streamwise regions become unstable. The latter explanation corresponds to observations in natural transition under the influence of freestream turbulence as discussed by Brandt and Henningson [2002]. In a third category the goal is to construct a low-order dynamical system of differential equations that display the elemental processes. All of the well regarded mechanisms have been verified by simplified experiments or DNS. While much of the information is for low Reynolds numbers, even there we are unsure of the importance of different self-sustaining mechanisms.

Panton [2005, 2007] also has reviewed the literature on the theory of wall turbulence from his viewpoint of composite expansions. Although the review begins with the work of the founding fathers, it includes modern high-quality experiments, direct numerical simulations and analyses that have been published this century. He feels the treatment offers a unifying way to think about wall turbulence. Based on scaling, he is able to redefine Townsend's [1961] active and inactive motion model. The essence is to say that the inactive part of the streamwise correlation $\overline{u^{2}}$ scales with $\left(\mathrm{u}_{\tau} \mathrm{U}_{\mathrm{c}}\right)$ and the active portion scales with $u \tau^{2}=\tau_{\mathrm{W}} / \rho$, the wall shear stress, as does the Reynolds shear stress $\overline{\mathrm{u}} \bar{v}$ [Panton, 2008]. As noted by Panton, mathematically the theory of turbulence is the leading term in asymptotic expansions for high Reynolds number with wall turbulence having two regions requiring two matched asymptotic expansions. A composite of these two expansions shows the effect of varying Reynolds number.

Bernard and Wallace [2002] summarize much on the structure of bounded turbulent flow for low pressure gradients and fully-developed channel flows and cover the basic analysis techniques. Analysis techniques were developed to elucidate specific structures that have been observed (or hypothesized) to exist in turbulent shear flows. They have reviewed a great deal of the observations by both experimental and computational means for what has become known as "coherent structures." Of course, the original research articles cited herein and in their text are the basic references that need to be studied for a detailed picture. But, for those desiring an introduction to the subject of coherent structures, we recommend their text (pages 152-176). There one will learn about low-speed sweeps, bursts, ejections, sweeps and shear layers. Often these structures were discovered by visual means. To study the structures more quantitatively, investigators wanted to analyze anemometry signals and this examination is where the analysis techniques come into play. Bernard and Wallace have integrated signal analyses nicely into their discussion of the structures observed.

Morrison [2007] has examined the interaction between inner and outer regions of turbulent wall-bounded flows. He uses high-Reynolds-number data (hence large $\mathrm{y}^{+}$) from the atmospheric surface layer and pipe flow to show that (1) the large scale motion penetrates to the wall, (2) the interaction is not a linear process and (3) it should be more generally accepted as an intrinsically non-linear one. 
Landahl (personal communication to $\mathrm{McE}$ ) at one time commented that streamwise vortices themselves were weak motions so they would not transfer much momentum. The long streamwise distance for a dye streak to develop is evidence that, if it is riding a vortex, that vortex is weak relative to the streamwise motion. More significant shear layers probably evolve when such vortices wobble against surrounding fluid, in the vertical or spanwise direction. Bradshaw [1994; Morrison and Westbury, 1996] suggested that a requirement for a structure to be "coherent" is that it should have a long persistence and so cannot carry a large fraction of the turbulence kinetic energy.

Adrian [2007] presents a useful review of ideas concerning coherent structures in wall turbulence, summarizing developments that occurred since 1991. He then concentrates on hairpin vortex organization as deduced primarily from DNS predictions and PIV measurements. Hairpins can occur singly or in packets, most commonly in the logarithmic layer. He notes that, while the growth of packets can provide a mechanism to transport momentum and tke, the transport cannot be exclusively due to this coherent structure. It is recognized that the very large scales of motion support a large fraction of the Reynolds stress - and the relationship between them and the hairpin packets remains to be established. While most of Adrian's experiments have dealt with the logarithmic layer and further out, the viscous layer would not be independent of the large eddies above it, e.g., Brodkey's sweeps. Adrian suggests that the outer eddies organize the inner eddies.

Guala, Hommema and Adrian [2006], say GHA, presented measurements for near fully-developed high-Reynolds-number turbulent flow in a circular pipe. These data cover the range $\sim 190<\mathrm{y}^{+}<\sim 8000$ and their "net force" results are for $\mathrm{y}^{+}>\sim 570$. At these locations volumetric entropy generation rates would be miniscule. The present manuscript concentrates on the viscous layer $\left(\mathrm{y}^{+}<\sim 30\right)$ for channels with $\mathrm{Re}_{\tau} \approx 150$ and 190. GHA interpret VLSMs (ery Large $\underline{\text { Scales of }}$ Motion) as the motions with $\mathrm{k}_{\mathrm{X}} \mathrm{R}$ $<2$ and find that, for their range, these motions contribute about 50-60 per cent of the Reynolds shear stress. However, this contribution decreases as the wall is approached and their spatial resolution prohibits accurate measurements in the viscous layer (their Fig. 7). GHA suggest that a very interesting topic for future work would be to explore the regions closer to the wall (p. 537 top). The spectral oil channel data of McEligot and Eckelmann [2006] would permit comparable study and analyses so we are initiating collaboration with Dr. Guala for that purpose; however, this new work is beyond the scope of the present study.

It would be desirable to examine the effects of lateral convergence (pressure gradient and streamwise acceleration) on the proposed models and coherent structures - but only a few characteristics can be studied with simultaneous time series data from a single X-probe plus a wall sensor. In our present study, we employ the analysis technique of conditional sampling and averaging to deduce effects on $\mathrm{S}_{\mathrm{t}}$,uy ${ }^{+}$and - as a by-product - are able to identify some modifications of structure behavior, primarily for sweeps or fronts and ejections. Particle image velocimetry systems can cover a more complete field and examine more aspects of hypothesized structures but usually lack the spatial resolution to treat the viscous layer well [Liu, Adrian and Hanratty, 2001].

Morrison and Westbury [1996] cite Bradshaw as suggesting in 1965 that splats set up a Stokes layer. They also credit him with describing ejections and sweeps as a form of ultimate eddy in that they are the most organized form of eddy and best able to maintain themselves against viscous dissipation while extracting energy from the mean flow - but they do carry almost all of the momentum.

\subsection{Conditional Sampling and Averaging}

Visual observations are the basis of descriptions of coherent structures. They have resulted in dynamic pictures of coherent motions that we now call the ejections and sweeps or fronts of interest in the present experiment plus many others. But we are not satisfied with just qualitative pictures or moving 
picture cartoons. We would like numbers that measure quantitatively what we see. The first simple efforts were when it was realized that the Reynolds stress from ejections or sweeps could be translated into four quadrants based on the sign of $\mathrm{u}$ and $\mathrm{v}$ and thus separated. This separation was simple enough to do with elementary circuits found in the literature. This approach became known as quadrant splitting and variations thereof. However, this technique did not turn out to be the precise technique desired to separate events in sub-categories. The addition of a window or threshold did help some, but not as much as desired.

To improve on the quadrant splitting, Vita [Blackwelder and Kaplan, 1976] and pattern recognition were invented. Vita was a first step in recognizing a specific pattern. It took many events and averaged them by superpositioning events at a single pin-point that was the maximum in the positive acceleration of the u-signal. Simple amplitude and other criteria could be used to refine the separation. Pattern recognition, which is similar, added additional criteria to allow the elucidation of the signal to occur faster and thus use many fewer events. In effect, it was more refined in extracting events. Some of added criteria were using multiple pin-points, normalization in time, and a temporal average for each event detected. This latter was important, since the local event near a wall could be different enough from the mean average across the flow, that the event changed sign and would occur in the wrong quadrant (wrong sign on uv).

The pattern recognition technique of Wallace, Brodkey and Eckelmann [1977], say WBE, seeks to account for the wide distributions of scales, amplitudes and frequencies seen in turbulent velocity signals. As they note, a flow structure passing a probe will only occasionally be intersected directly through its center; often it will be intersected near an edge. In addition, structures will pass the probe at different stages of their lifetimes. Accordingly, the PR technique was successfully developed to detect the whole range of sizes and to normalize them to obtain meaningful average patterns. It is based on the observation of many time traces. WBE were convinced that a characteristic pattern related to the coherent-structure sequence occurs and its predominant feature appears in the streamwise fluctuating velocity signal $\mathrm{u}\{\mathrm{t}\}$. The feature is a weak deceleration of the flow, followed by a period of almost constant and relatively low velocity and then a strong acceleration shown by a large positive temporal gradient in $\mathrm{u}\{\mathrm{t}\}$. Details of the approach and some characteristics of the patterns observed in an lpg channel flow are presented in the original paper [WBE] with further improvements explained in a later paper [Brodkey et al., 1985].

Using Vita in a turbulent boundary layer, Blackwelder and Kaplan [1976] conditionally sampled $\mathrm{u}\{\mathrm{t}\}$ traces with detection based on identification of highly energetic fluctuations of $\mathrm{u}$. Their conditionally-averaged signal exhibited the same characteristics as the PR approach: a weak deceleration followed by a strong acceleration. WBE concluded that the PR and Vita techniques detect the same aspect of the overall "bursting phenomenon." As shown by Eckelmann and Wallace [1981], the Vita detection time $\mathrm{t}^{+}=0$ - when sampling $\mathrm{u}\{\mathrm{t}\}$ - corresponds to the maximum positive temporal slope in $<u^{+}>$.

However, let us not forget that the basis of all of this development is still the visual observation. Based on the observations, these attempts and others have been made to extend the interpretations of the apparent motions to develop simple criteria to be able to classify the various events from examination of temporal measurements with thermal anemometry. As indicated above, techniques for conditional-sampling and -averaging of time series data in channel and boundary layer flows have been developed as by Wallace, Eckelmann and Brodkey [1972], Willmarth and Lu [1972], Lu and Willmarth [1973], Blackwelder and Kaplan [1976], Wallace, Brodkey and Eckelmann [1977] and Eckelmann, Wallace and Brodkey [1978] and others. Excellent reviews and/or analyses of these techniques have been given by Eckelmann and Wallace [1981], Blackwelder and Haritonidis [1983], Johansson and Alfredsson [1983], Alfredsson and Johansson [1984], Bogard and Tiederman [1986], Luchik and Tiederman [1987], Morrison, Tsai and Bradshaw [1989], Morrison and Bradshaw [1989], Morrison, Subramanian and 
Bradshaw [1992], Wallace and Foss [1995], Andreopoulos and Agui [1996] and Honkan and Andreopoulos [1997] and the reader is referred to these papers for further details. However, it is appropriate to indicate key observations which relate to the present study.

As shown by Eckelmann and Wallace [1981], the Vita detection time $\mathrm{t}^{+}=0$ corresponds to the maximum positive slope in the conditionally-averaged $\left\langle\mathrm{u}^{+}>\right.$signal. They employed the data of Blackwelder and Kaplan to show that the maximum value of $(\partial \tilde{u} / \partial y)$ occurs at the same time as the maximum of $(\tilde{\partial u} / \partial t)$. Further, they demonstrated that, with the pattern recognition scheme of Wallace, Brodkey and Eckelmann [1977], $(\partial \mathrm{u} / \partial \mathrm{y}) \max$ also occurs when $(\partial \mathrm{u} / \partial \mathrm{t})$ peaks. Therefore, either of these conditional averaging schemes would be reasonable for examining the maximum temporal values of the entropy generation rate. With a two-sensor gradient probe, Randolph, Eckelmann and Nychas [1987] demonstrated that $(\tilde{\partial u} / \partial \mathrm{y})$ and $(\tilde{\partial u} / \partial \mathrm{t})$ are highly correlated and, therefore, can serve as criteria for the recognition of sweeps in an Eulerian coordinate system. They concluded that these calculations confirm the visual studies of Corino and Brodkey [1969] and Nychas et al. [1973] showing that large spatial gradients always occur together with a large temporal gradient.

For their turbulent boundary layers, Blackwelder and Haritonidis [1983] discussed the advantages and disadvantages of probe measurements compared to visual observations and described the Vita technique. They indicated that large excursions are associated with accelerations and decelerations of the streamwise velocity signal. They cited Chen and Blackwelder as reporting that the more important aspects of their bursting phenomenon were primarily due to the accelerations so they added the criterion that $\mathrm{du}\{\mathrm{t}\} / \mathrm{dt}$ be positive. They concluded that their bursting frequency $\mathrm{fb}$ was a weak function of $\mathrm{y}$ but no region could be identified where $\mathrm{f}_{\mathrm{b}}$ was independent of the Vita threshold. They further concluded that the bursts detected by Vita correlate well with large (uv) and fb scales with wall variables.

Bogard and Tiederman [1986] conducted a useful review of conditional sampling techniques, examining the capabilities of the different techniques to identify flow visualized by traces corresponding to their definition of ejections. Their burst was defined as the occurrence of a group of closely-spaced ejections that are directly associated with the breakup of a single visually-observed streak. Their study showed the difficulties of identifying ejections and bursts by different techniques, the differences between the techniques and their sensitivities to their thresholds. They demonstrated that their Q2 approach is a good method to detect ejections as defined by the characteristics of their visually-observed dye traces.

Luchik and Tiederman [1987] provided a good literature review and examined the performance of various sampling schemes for the probability of detecting the events they defined. (They are among the few investigators who have noted that the pressure gradient is inherently substantial in low-Reynolds-number channel flow.)

Alfredsson et al. [1988] showed that for a zpg boundary layer the temporal correlation between the streamwise velocity fluctuation and the fluctuating wall shear stress was almost unity in the linear layer and about 0.8 near $\mathrm{y}^{+}=15$, a common location for sampling with a hot wire or hot film probe. They concluded that the instantaneous velocity profile is also linear through the (mean) linear layer.

Morrison, Tsai and Bradshaw [1989] cite Luchik and Tiederman [1987] as noting that sampling the streamwise fluctuation $\mathrm{u}\{\mathrm{t}\}$ with the Vita technique detects the large excursions where its gradient is largest; MTB indicate that with a short averaging period $\mathrm{T}^{+}$it becomes a "pure gradient criterion." They compared various Vita and QS approaches as applied to their data for the logarithmic layer of high-Reynolds-number TBLs and suggest that the threshold should be based on $\mathrm{u}_{\tau}$. 
For a zpg boundary layer, Morrison and Bradshaw [1989] examined the instantaneous wall shear stress when conditioned on ejections or sweeps (as defined by their algorithm) directly above the wall sensor. The "wall" sensor was a surface hot wire at $\mathrm{y}^{+} \approx 3-1 / 2$ and probe locations appear to be at $\mathrm{y}^{+}$ greater than about 250 or so. They suggest that their information is relevant to development of a wall boundary condition for time-dependent simulations of turbulence. For sampling, their "Vita plus level" approach is applied to the instantaneous uv normalized via $\overline{\mathrm{u}} \overline{\mathrm{v}}\{\mathrm{y}\}$. They note that ensemble-averaged signatures of uv, strain rate and vorticity are very similar to Falco's "typical eddy." Their sweeps induce a high value of wall shear stress while their ejections reduce it. They interpret their Figure 11 as showing that inertial structures associated with ejections conform with universal inner scaling while sweeps produce large-scale inactive motion at the wall. The "splatting" motion described by Moin and Kim [1982] is caused by large-scale sweeps and is responsible for transfer of energy from the v-component motion to the $\mathrm{u}$ and $\mathrm{w}$ components at $\mathrm{y}^{+}<10$, causing extra direct dissipation in the sublayer. The contribution of large-eddy sweeps to $\mathrm{u}_{\tau}$ can be significant.

Morrison, Subramanian and Bradshaw [1992] measured temporal vorticity components, strain rates and Reynolds shear stress for $\mathrm{y}^{+}>250$ in high-Reynolds-number turbulent boundary layers with negligible pressure gradients. Conditional sampling was via a modified Vita technique. They define ejections as a short region of intense turbulence activity with $\mathrm{u}<0$ and $\mathrm{v}>0$ and described them as the centers of inertial eddies like Townsend's attached eddies [1961] with a strong correspondence to visually observed bursts. They suggest that ejections are the primary control of the inner-outer interactions. Bursts are defined as the complete sequence of outward moving fluid and sweeps are motions with $\mathrm{u}>0$ and $\mathrm{v}<0$. Sweeps might be described as inverted mushrooms. They observe that negative spanwise vorticity dominated their ejections with positive dominating their sweeps. They operationally define ejections and sweeps by the quadrants and their intermittency $\mathrm{I}\{\mathrm{t}\}=1$ (from their eqn $3.1 \mathrm{a}, \mathrm{b}$ the detection criterion $\mathrm{TH}$ essentially determines the "length" [duration times local $\mathrm{U}\{\mathrm{y}\}$ ] of the event). The major difference between ejections and sweeps, or the inertial structures associated with them, is that the former conform to Townsend's attached eddies while the latter correspond to his inactive motion. Ejections control the interaction between inner and outer layers. Sweeps are large-eddy 'centres' which control the distribution of shear stress along with ejections and produce large low-wave-number fluctuations in the motion along the wall. Low-wave number energy is transported to the wall by large-scale inertial eddies associated with sweeps (usually referred to as "splats") which cause extra direct dissipation in the sublayer. In terms of Bradshaw's interpretation or extension of Townsend's model, there are two related sources of inactive motion, both principally caused by splats. The first source is low-wavenumber velocity fluctuations in the inner layer caused by these large eddies. The second is pressure fluctuations at $\mathrm{y}^{+}<30$ caused by the splatting mechanism by which v-component energy is transferred to the two horizontal components.

Wallace and Foss [1995] presented a nice review of measuring techniques and their success in determining velocity gradient fluctuations, mostly by thermal anemometry. Their comparison of $\left(\omega_{z}{ }^{\prime}\right)^{+}$ data (primarily $\partial \mathrm{u}^{+} / \partial \mathrm{y}^{+}$) showed considerable scatter, particularly in the viscous layer. (The symbol represents streamwise vorticity and the prime indicates a root-mean-square value.) They also pointed out the mistake of using Clauser plots to deduce $u_{\tau}$ for the calculation of $\left(\omega_{\mathrm{Z}}\right)^{+}$.

Andreopoulos and Agui [1996] discussed wall vorticity measurements and some related literature. For a zpg boundary layer in a wind tunnel they deduced the vorticity flux at the wall from an array of pressure transducers. Based on their assumptions, they explain the relations of ejections and sweeps to the sign of $\left(\partial \omega_{\mathrm{Z}} / \partial \mathrm{y}\right)$ (their p. 71). They extended their conditional sampling technique to detect the peak value of the $\left(\partial \omega_{\mathrm{X}} / \partial \mathrm{y}\right)$ signal during the time it is above a threshold level. Although their measured event rate $\mathrm{f}^{+}$is a rather strong function of the threshold level, the deduced flow patterns are much less dependent. As the threshold increases, stronger events contribute more to the ensemble-average pattern 
and some features become more pronounced. They observed that the major feature of the vorticity flux appeared to be a change of sign of $\left(\partial \omega_{\mathrm{Z}} / \partial \mathrm{y}\right)$ at the detection based on $\left(\partial \omega_{\mathrm{X}} / \partial \mathrm{y}\right)$ and interpreted this observation in terms of their models of vortex behavior. From these wall measurements they are able to infer considerable temporal behavior within the flow. In particular, they suggest that sweeps, which are usually characterized as high-momentum outer-layer fluid moving towards the wall, can be considered as an impulse of a jet-like fluid motion splashing on the wall. Their Figure 30 describes such a conceptual model and its scenario whereby ejections and sweeps are formed.

Honkan and Andreopoulos [1997] conducted a nice, extensive experiment with a nine-wire probe of diameter $\mathrm{D}^{+} \approx 28$ or so. Conditional sampling was accomplished with a special method of detection, differing from the common techniques. Their data for production and dissipation appear reasonable for $\mathrm{y}^{+}>\sim 40$ but diverge in the viscous layer, as might be expected. Uncertainty in the measurement of $\partial \mathrm{u} / \partial \mathrm{y}$ is discussed.

Wilhelm, Härtel and Eckelmann [1998] showed that the average shear layer obtained from their Visa procedure agreed very well with the inclined shear structure computed from two-point spatial correlation functions.

It is appropriate to indicate key observations from the examinations of sampling that relate to or guide the present study: Ultimately, conclusions from conditional sampling will be qualitative for many aspects. Different investigators define the same structures variously and use conditional sampling techniques differently, so it is important to specify the present definitions and usage. In this study, we define sweeps and (abrupt) ejections as observed by Corino and Brodkey. If employed intelligently, one can use conditional sampling and averaging to deduce some characteristics of inclined shear layers (as with sweeps) and ejections. The Vita detection time corresponds to the maximum positive slope in the temporal signal, e.g., $\mathrm{du} / \mathrm{dt}$. The maximum value of $(\tilde{\partial \mathrm{u} / \partial \mathrm{y}})$ occurs at the same time as the maximum of $(\partial \tilde{u} / \partial t)$. Further, with the pattern recognition scheme $(\partial \mathrm{u} / \partial \mathrm{y}) \max$ also occurs when $(\partial \mathrm{u} / \partial \mathrm{t})$ peaks. Therefore, either of these schemes would be reasonable for examining the maximum temporal values of the entropy generation rate.

While Vita and PR detect fronts as in the sweeps described by Corino and Brodkey, the Q2 approach can be a good method to detect ejections. As the threshold of QS is raised with Q2 detection, the structures identified approach the abrupt ejections of Corino and Brodkey rather than the minor outward flows of some dye trace visualization. Measured event rates can be rather strong functions of the threshold levels, but the deduced flow patterns are much less dependent on threshold. Though these deduced flow patterns may be somewhat qualitative, relative timing between the events observed near a detection can be considered to be quantitative.

\subsection{Conspectus}

The experiment and analysis procedures are next presented briefly. In order to accomplish the present objective of determining whether mean pressure gradients affect the temporal behavior of entropy generation, the time series data from the oil channel experiments of ME have been synchronized and analyzed. One wonders as well how the coherent structures of canonical flows at lpg are affected by a significant streamwise pressure gradient. Our observations are examined for effects on (1) structure event rates, (2) ejections via quadrant splitting and, in the vicinity of the sweeps, (3) ensemble-averaged measurements of $\mathrm{u}^{+}, \mathrm{v}^{+}(\mathrm{uv})^{+}, \tau_{\mathrm{W}}{ }^{+}$and of (4) the dominant contribution to the instantaneous entropy generation rate. We summarize with a few concluding remarks. As with the measurements of Morrison and Westbury [1996] the insights afforded by the present results may also have significance to (1) the 
active control of wall turbulence and (2) development of wall boundary conditions in large eddy simulations (LES). 


\section{EXPERIMENT AND ANALYSIS PROCEDURES}

The equipment, procedures, experimental uncertainties and time series measurements employed in the present investigation are the same as those presented by McEligot and Eckelmann [2006]. The data were obtained with X-probes and a wall surface-probe using hot film sensors in the oil channel at the Max Planck Institut für Strömungsforschung. The channel was $22 \mathrm{~cm}$ wide and $1 \mathrm{~m}$ deep with a length of $8 \mathrm{~m}$. Measurements examined two general situations: (1) a favorable streamwise pressure gradient induced via the lateral convergence of a two degree ramp installed on the floor of the channel and (2) approximately fully-developed flow as in the earlier studies of Eckelmann and colleagues [Eckelmann, 1974; Wallace, Eckelmann and Brodkey, 1972; WBE; Blackwelder and Eckelmann, 1978; Kreplin and Eckelmann, 1979a; etc., etc.] for comparison purposes. Further understanding and specific details concerning the experiment are provided in the papers of Eckelmann [1974] and ME plus a technical report by McEligot and Eckelmann [2003].

Only one X-probe was used at the measuring station in order to avoid the mutual interference between signals that can occur when employing a "rake" of multiple sensors. However, since the time series for each run included the wall signal simultaneously with the signals from the two sensors of the $\mathrm{X}$-probe, it is possible to relate the signals at the various wall-normal locations $\left(\mathrm{y}^{+}\right)$via conditional sampling. Thus, one may form simultaneous ensemble averages for a range of $\mathrm{y}^{+}$without having additional probes to disturb the flow, i.e., one can construct the equivalent of two-component signals from a rake as by Blackwelder and Kaplan [1976] without actually having a rake of X-probes. This approach is comparable to that of Johansson, Alfredsson and Eckelmann [1987] in examining the spatial distributions around an inclined shear layer in a lpg channel flow. For the hpg measurements at $\mathrm{y}^{+} \approx 16$, the estimated

experimental uncertainty of $<\partial \tilde{\mathrm{u}}^{+} / \partial \mathrm{y}^{+}>$is of the order of 0.01 and for the resulting $\left\langle\mathrm{S}_{\mathrm{t}, \mathrm{uy}}{ }^{+}>\right.$is about 0.009 , both in non-dimensional wall units. (It does not make sense to present per cent uncertainties because $<\tilde{\partial \tilde{u}} / \partial \mathrm{y}>$ in the denominator can pass through zero.) For the lpg data at $\mathrm{y}^{+} \approx 13$, these uncertainties are of the order of 0.013 and 0.012 , respectively.

Initially, three conditional-sampling techniques were used to examine the temporal behavior of key coherent structures: (1) for ejections, the quadrant-splitting approach of Wallace, Eckelmann and Brodkey [1972] and Lu and Willmarth [1973] and, for sweeps, (2) the Vita analysis of Blackwelder and Kaplan [1976] and (3) pattern recognition as by WBE. Each has advantages so different techniques can be applied for different purposes in examining temporal features of turbulence structure. Calculations are accomplished via a Fortran program developed by WBE as extended by Brodkey et al. [1985] and then modified slightly for the present work (to accommodate different sensor calibration approaches and to replace a five-sensor probe with an X-probe, the wall sensor and an upstream reference probe).

Johansson, Alfredsson and Eckelmann [1987] used the Vita technique and simultaneous measurements from two sensors to construct the temporal and, hence, spatial fields in the vicinity of internal shear layer passage. Their constructed spatial field shows maximum values of $\partial \mathrm{u} / \partial \mathrm{x}$ and $\partial \mathrm{u} / \partial \mathrm{y}$ at detection (passage). By applying their Visa technique, which detects the passage of inclined internal shear layer structures, Johansson, Alfredsson and Kim [1991] were able to construct comparable spatial and temporal behavior of $<\mathrm{u}>,<\mathrm{v}>,<\mathrm{uv}>,<\mathrm{p}>$ and turbulence production in the vicinity of this event from DNS of channel flow with a slight to moderate streamwise pressure gradient. Their conditionallyaveraged spatial fields in the $x-y$ plane provide useful insight for interpreting the present Vita deductions for lpg. While Visa essentially triggers on high values of instantaneous $\partial \mathrm{u} / \partial \mathrm{x}$ (analogous to the $\partial \mathrm{u} / \partial \mathrm{t}$ of Vita), their Figures 4 and 5 demonstrate that $\partial \mathrm{u} / \partial \mathrm{y}$ is considerably greater than $\partial \mathrm{u} / \partial \mathrm{x}$ there. It is $\partial u / \partial y$ that is expected to be a dominant contributor to temporal entropy generation. 
For this paper a few defining comments on the analysis techniques are in order. One can consider detection as occurring when a signal exceeds a value given by a specified number times a "normalizing factor." The Vita detection was applied to the time series for the streamwise fluctuation u. As by Blackwelder and Haritonidis [1983] the conditions for detection were that the variance defined by Blackwelder and Kaplan must exceed the quantity $\mathrm{k} \cdot\left(\mathrm{u}^{\prime}\right)^{2}$ (where $\mathrm{u}^{\prime}$ represents the root-mean-squared value of the fluctuation $\mathrm{u}$; likewise for $\mathrm{v}$ ) and the fluctuation must be increasing. The constant $\mathrm{k}$ served as threshold control and, by adjustment, determined the number of events detected. This detection corresponds to the timing of "fronts" passing the probe location [Kreplin and Eckelmann, 1979b] or, in the interpretation of others, identifies an ejection/sweep interface in the time series. As noted earlier, Vita detection is triggered by maximum values of du/dt which correspond to the desired maximum $(\partial \tilde{u} / \partial y)$.

The quadrant-splitting technique (QS) aims at spotting the ejections described by Corino and Brodkey [1969] for their flow visualizations of pipe flow. In the QS classification, quadrants are defined in terms of the global overall averages, i.e., as fluctuations relative to the time-mean components $\mathrm{U}$ and $\mathrm{V}$ determined by integration of the entire time series. Requirements for detection are that the peak value of the instantaneous $|-u v|$ exceed a quantity HS $\cdot u^{\prime} v^{\prime}$. If the wall normal fluctuation $v$ is required to be away from the wall, it may be considered to be a second quadrant detection (Q2) with its threshold controlled by the constant HS. In the process of examining the time series, all individual samples are categorized by their quadrants relative to the mean velocity and their "instantaneous Reynolds shear stress" or turbulent momentum transfer rate is determined. A Q2 event corresponds to a decelerating outward motion and a Q4 event to an accelerating wallward motion but, as noted earlier, not necessarily to abrupt ejections [Corino and Brodkey, 1969] or sweep fronts [Kreplin and Eckelmann, 1979b]. By choosing a sufficiently high threshold, QS detections can be limited to the abrupt ejections of Corino and Brodkey.

The pattern recognition process of Wallace, Brodkey and Eckelmann [1977; Brodkey et al., 1985] overcomes some difficulties of other conditional-sampling approaches and tends to detect sweep front passage. In contrast to QS and Vita techniques, PR determines a short-term temporal (floating) average, called TPAV, for each specific event identified. It aims at accounting for shape and size of the transient events via a criterion for a signal slope (versus time) comparison ratio and the requirement that the maximum fluctuation $\Delta \mathrm{u}_{\text {peak }}$ for the event exceed a specified quantity "AFACT" (as in amplitude factor) times the difference $(\tilde{u} \max -\tilde{u} \min )$ calculated for the entire time series. This amplitude criterion was added to the computer program subsequent to the original publication by WBE and, along with other modifications, is explained by Brodkey et al. [1985]. Adjustment of the required slope ratio and the constant AFACT establish the threshold for the sampling. PR also has the advantage of normalization of events to elucidate wave or event forms with few events required. As shown by Eckelmann and Wallace [1981], when PR is applied to the $\tilde{\mathrm{u}}$ signal, detection is also triggered by maximum values of $\tilde{\mathrm{du}} / \mathrm{dt}$ which correspond to the desired maximum $(\tilde{\partial u} / \partial y)$.

With each technique, once the sampling criteria are satisfied at an instant, all time series may be ensemble-averaged about this time. Thus, temporal ensemble-averaged traces for the two velocity fluctuations and the wall shear stress are related to the time of detection. 


\section{RESULTS}

McEligot and Eckelmann [2006] provided mean turbulence statistics in the viscous layer at $\mathrm{y}^{+} \approx 5,7$, 10,15 and 25 (nominal values) for four sets of operating conditions, two with favorable streamwise pressure gradients and two for the corresponding fully-developed flows. As noted, the present objectives are to determine whether streamwise mean pressure gradients affect the temporal behavior of entropy generation rates in the viscous layer and, if so, which aspects are affected significantly. In the present results we concentrate on the two extreme cases: $-\mathrm{K}_{\mathrm{p}} \approx 0.008$ for fully-developed flow and $-\mathrm{K}_{\mathrm{p}} \approx 0.020$ (near laminarizing) for laterally-converging flow. We conduct the conditional sampling for three wall distances $\mathrm{y}^{+} \approx 7,15$ and 25 in order to represent temporal behavior near the wall, in the region of greatest turbulence production and near the edge of the viscous layer, respectively.

\subsection{Turbulent Structure Event Rates}

From different evidence Kline et al. [1967], CMM and Finnicum and Hanratty [1988] concluded that an effect of a favorable streamwise pressure gradient is to reduce an apparent bursting rate. These observations may be examined in terms of the various turbulent structures involved. One might expect that, with constant values of the threshold controls, the measured event rates may be compared across the viscous layer for the two extremes of pressure gradients. Since the techniques have different scaling factors, the magnitudes should not be compared but their trends might be.

For these comparisons, the key parameters for the pattern recognition algorithm (PR) have been specified as AFACT $=0.4$ with "single point pinning" and a multiplier of 1.5 for the slope comparison ratio. For Visa, the averaging window has been adjusted to $\left(\mathrm{T}_{\mathrm{avg}}\right)^{+} \approx 10$ with $\mathrm{k}=0.32$ for the threshold. These two approaches are believed to detect sweeps. The quadrant splitting subroutine can be programmed to detect apparent sweeps or ejections or both. The threshold HS for QS was 4.35 and the resulting data were further classified as Q2 or Q4; at this threshold they can be considered to represent the abrupt ejections and sweeps of Corino and Brodkey, respectively. Other secondary control parameters and constraints were also held fixed. The total sampling time was the same for all runs.

\subsubsection{Reference Condition - Low Pressure Gradient}

The trends of ejection and sweep rates for canonical flows are consistent with earlier observations which have been extensively reported in the literature. However, further physical explanations of this behavior and apparent differences are in order.

It would be logical that, very close to the wall, one would see a reduction in rate of ejections. But as explained in the Introduction, over the rest of the layer, the induced ejection rate should be constant since they are part of the same event sequence no matter where they are deduced. In Figures 1a and $1 \mathrm{~b}$ only the pattern recognition events have the expected shape; their rates rise from a low value at the wall and become approximately constant away from the wall.

Measurements of the numbers of sweeps and ejections detected are presented in Figure 1a while non-dimensional event rates $\mathrm{f}^{+}\left(=\mathrm{fv} / \mathrm{u} \tau^{2}\right.$ where $\mathrm{f}$ is the number of events per unit time identified at a point) are provided in Figure 1b. Use of wall coordinates accounts for some variation with Reynolds number and perhaps pressure gradient. Here the data symbols are connected by solid lines for the high pressure gradient (hpg) case and by dashed ones for lpg (low pressure gradient). For the sweeps determined by both Vita and PR, one sees a tendency for the detection rate to increase with distance from the wall. However, the number of sweeps identified by the QS algorithm - based on the uv product rather than the streamwise fluctuation $\mathrm{u}$ - appears to decrease with distance from the wall; fewer exceed the $\mathrm{u}^{\prime} \mathrm{v}^{\prime}$ 
threshold. Since the thresholds are defined in physical variables here (rather than wall coordinates), the required magnitude variations to trigger sampling of the signals differ. For example for the hpg run, the QS normalizing factor ' $\mathrm{u}^{\prime} \mathrm{v}$ ' is approximately equal to $0.35,0.61$ and $0.68 \mathrm{~cm}^{2} / \mathrm{sec}^{2}$, increasing successively, for the three locations examined $\left(\mathrm{y}^{+} \approx 7.4,16.0\right.$ and 26.4) while the corresponding values of the normalizing factor for Vita are $\left(\mathrm{u}^{\prime}\right)^{2} \approx 3.2,5.2$ and $3.8 \mathrm{~cm}^{2} / \mathrm{sec}^{2}$, increasing then decreasing for the same locations. It could be interesting to define the sampling criteria in appropriate wall variables as suggested by Morrison, Tsai and Bradshaw [1989] but such a study is beyond the present scope.

\section{Events across viscous layer}

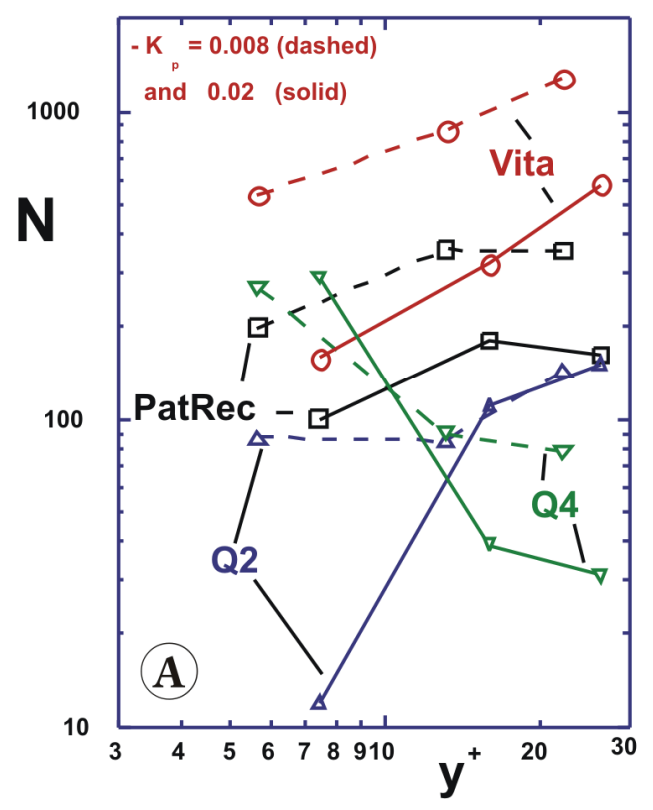

Event rates across viscous layer

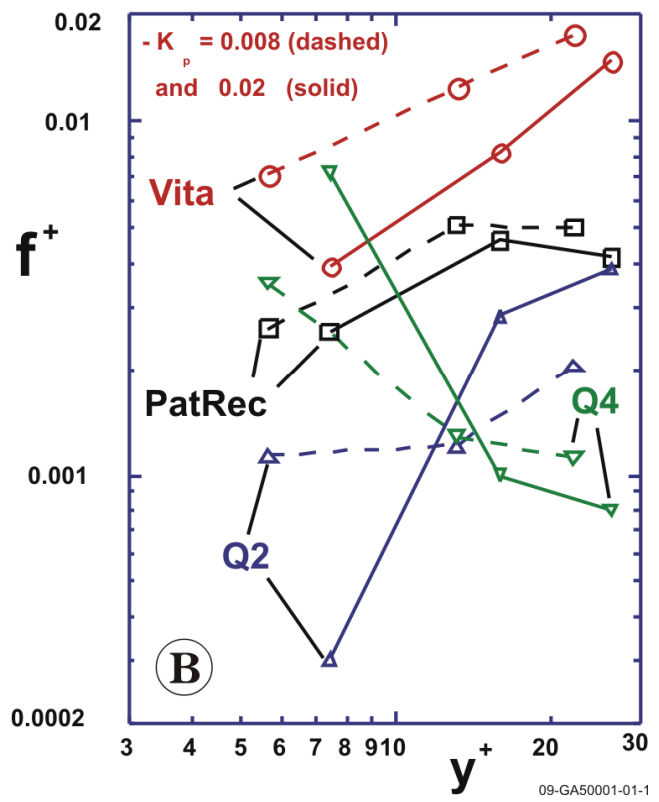

Figure 1. Sweeps and ejections identified across the viscous layer non-dimensionalized with wall coordinates from conditional sampling by the Vita technique (sweeps, circles) [Blackwelder and Kaplan, 1976], by pattern recognition, called PatRec (sweeps, squares) [Wallace, Brodkey and Eckelmann, 1977] and by quadrant splitting (triangles) [Wallace, Eckelmann and Brodkey, 1972]: (a) totals identified and (b) non-dimensional rates. Symbol Q2 represents ejections and Q4 sweeps; solid lines connect data at the higher streamwise pressure gradient $\left(-\mathrm{K}_{\mathrm{p}} \approx 0.02\right)$ and dashed ones are for the lower pressure gradient ($\left.\mathrm{K}_{\mathrm{p}} \approx 0.008\right)$.

The ejection rate from QS generally increases with distance from the wall. With the present level of the QS threshold criterion, significantly more ejections are detected than sweeps away from the wall while the reverse is true near the wall. WBE indicate that ejections originate somewhat further from the wall and move outwards while sweeps can and do come right up to the wall. The lpg results for QS are consistent to some extent with earlier observations from several viewpoints: visual [Corino and Brodkey, 1969], u, v and uv probability density functions (pdfs) [Brodkey, Wallace and Eckelmann, 1974], pattern recognition [WBE] and instantaneous "production" [Eckelmann et al., 1977] - but these trends with $\mathrm{y}^{+}$ are not obvious in the joint pdfs of Wallace and Brodkey [1977]. Blackwelder and Haritonidis [1983] concluded that their bursting frequency $\mathrm{fb}_{\mathrm{b}}$ was a weak function of $\mathrm{y}$ but no region could be identified where $\mathrm{fb}_{\mathrm{b}}$ was independent of the Vita threshold. However, since the threshold was the same for all their data, the relative variation of frequencies reported cannot be attributed to thresholds. They further concluded that the bursts detected by Vita correlate well with large (uv) and fb scales with wall variables. 
For Vita and QS the turbulent structure rates vary with distance from the wall. It is recognized that variation of the control parameters will change the numbers of events detected in a given time series. It is further recognized that, between the techniques, the normalizing quantities vary differently with respect to pressure gradient and wall distance. Both $\left(\mathrm{u}^{\prime}\right)^{+}$and $\left(\mathrm{v}^{\prime}\right)^{+}$are functions of $\mathrm{y}^{+}$but, as predicted by Spalart [1986] and shown by ME, $\left(v^{\prime}\right)^{+}$is more sensitive to a favorable pressure gradient. Consequently, the normalizing quantity u'v' in the threshold for quadrant splitting can be expected to change more with pressure gradient than $\left(\mathrm{u}^{\prime}\right)^{2}$ and $\left(\tilde{\mathrm{u}}_{\max }-\tilde{\mathrm{u}}_{\min }\right)$ in Vita and pattern recognition, respectively. (In later comparisons, thresholds have been adjusted for some runs to give approximately the same number of detected events across the viscous layer for ensemble averaging.)

With knowledge of some details of the detection technique and of typical time series across the viscous layer, one can provide a plausible explanation of the trend of sweeps identified by Vita versus $\mathrm{y}^{+}$. If the sweeps and ejections pass across most of the viscous layer as suggested by Corino and Brodkey, then for a given flow one would expect the detections/time to be approximately invariant with $\mathrm{y}$. In this technique the threshold criterion is normalized by the local value of the mean square fluctuation; i.e., the calculated signal must exceed $\mathrm{k} \cdot\left(\mathrm{u}^{\prime}\right)^{2}$ to define a sweep. For our lpg experiment $\left(\mathrm{u}^{\prime}\right)^{2} \approx 4.2,9.2$ and $7.8 \mathrm{~cm}^{2} / \mathrm{sec}^{2}$ for $\mathrm{y}^{+} \approx 5.6,13.2$ and 22.3 , respectively. So in the outer part of the viscous layer the required threshold level increases approaching the wall. Vita does not utilize u directly but rather a difference from a moving average - so it detects "rapid" variations from the moving average, not the level of $\mathrm{u}$. It is responsive to high values of the instantaneous $\partial \mathrm{u} / \partial \mathrm{t}$. The time series presented by Eckelmann [Figures. 18-21, 1970] for $\mathrm{u}\{\mathrm{t}\}$ show that, as $\mathrm{y}^{+}$increases across the viscous layer, more small-scale rapid fluctuations appear in the signal. These fluctuations can introduce significant $\partial \mathrm{u} / \partial \mathrm{t}$ although only for brief intervals. So the rate of sweep detections should increase with y (even if the threshold were not decreasing slightly).

In the QS technique the algorithm searches for instantaneous values of uv that are greater than the threshold criterion. From traces of uv $\{\mathrm{t}\}$ [Eckelmann, Fig. 28, 1970], one sees that the magnitudes and numbers of "significant excursions" increase as $\mathrm{y}^{+}$varies from three to twenty. So if one had a constant threshold, such as HS $\bullet \mathrm{u}^{2}$, the detected numbers of QS events would likely increase with $\mathrm{y}^{+}$in the viscous layer. However, in the present QS subroutine the threshold criterion is normalized by the product $u^{\prime} v^{\prime}$ so the instantaneous values of uv must exceed HS $\bullet\left(u^{\prime} v^{\prime}\right)$ to identify sweeps or ejections. For our lpg experiment $\left(\mathrm{u}^{\prime} \mathrm{v}^{\prime}\right) \approx 0.46,1.2$ and $1.7 \mathrm{~cm}^{2} / \mathrm{sec}^{2}$ for $\mathrm{y}^{+} \approx 5.6,13.2$ and 22.3 , respectively. The consequent variation of the threshold counters the trend of increased "excursions" and, for example, the lower value of u'v' near $\mathrm{y}^{+}$of five allows more events to be validated there. These identifications can be either ejections or sweeps, depending on the sign of $\mathrm{v}$ (in the present processing).

As noted, high values of the threshold multiplier HS, such as the current 4.35, will limit the identifications to only the most "energetic" events, corresponding to the abrupt ejections described by Corino and Brodkey (with the further requirement that $\mathrm{v}>0$ ). For qualitative insight into the question why more ejections may be detected towards the edge of the viscous layer one can consider a phenomenological description as in some explanations of Reynolds analogy [Kays, 1966]. For ejections outward from the low velocity region, if their x-momentum remains approximately constant, the magnitudes of their fluctuations $\mathrm{u}=\tilde{\mathrm{u}}-\mathrm{U}$ will increase as they move towards the edge of the viscous layer where the local mean $U$ is nearer to that of the "core" or the freestream. Thus, the magnitude of the fluctuation uv becomes greater in the outer region of the viscous layer and it is more likely to exceed the QS threshold than when back in the environment where it originated. (Comparable ideas have been discussed in the context of transition as "lift-up effect," low-speed streaks, "backward jets," "blips," 
negative "spikes" and such [Liepmann, 1943; Landahl, 1980; Goldstein and Leib, 1993; Kendall, 1998; Wu et al., 1999; Jacobs and Durbin, 2001; Wundrow and Goldstein, 2001].) Equivalent reasoning will also qualitatively explain an increase in sweeps as the wall is approached. Consequently, the QS algorithm is more likely to detect sweeps near the wall and ejections towards the outer edge of the viscous layer.

\subsubsection{Effects of Pressure Gradient}

For both pressure gradients the trends with wall distance are essentially the same. When examined as numbers of events $\mathrm{N}$ detected at a point (Figure 1a), the data representing sweeps generally show reductions as the pressure gradient is increased. In the outer part of the viscous layer the number of ejections detected is approximately the same for both pressure gradients. However, when the event rates are treated in terms of wall coordinates $\left(\mathrm{f}^{+}\right)$the conclusions differ; some of the differences again can relate to the scaling of thresholds.

If the wall behavior for event rates at a point should scale with wall variables, as velocities, distances and time do without the effect of a pressure gradient, then one would expect that an appropriate basis for thresholds could be the same values in wall coordinates. For example, in sweep detection by Vita, using the same value of $\mathrm{k} \cdot\left(\mathrm{u}^{\prime 2}\right)^{+}$at the same location $\mathrm{y}^{+}$, would conceptually yield the same value of $\mathrm{f}^{+}$there. Since the computer algorithm uses physical values of $\mathrm{u}^{\prime}(\mathrm{cm} / \mathrm{sec})$, the same non-dimensional threshold would require setting $\mathrm{k}_{2}=\mathrm{k}_{1} \bullet\left(\mathrm{u}_{\tau 2} / \mathrm{u}_{\tau 1}\right)^{2}$ for the calculations at the second condition. If $\mathrm{u}_{\tau 2}<\mathrm{u}_{\tau 1}$ (as our hpg is relative to the lpg results), $\mathrm{k}_{2}$ should be lower than $\mathrm{k}_{1}$. If $\mathrm{k}$ is held the same as is done in our comparisons, the threshold would be effectively higher in the second case - giving fewer detections $\mathrm{N}$ per unit time; but since $\mathrm{N}$ is not linearly related to $\mathrm{u} \tau$, the conversion to $\mathrm{f}^{+}$may counter, but would not necessarily cancel, the effect of the higher threshold. The Vita and PR algorithms utilize the time series $\mathrm{u}\{\mathrm{t}\}$ for detection. As mentioned, ME and others found $\left(\mathrm{u}^{\prime}\left\{\mathrm{y}^{+}\right\}\right)^{+}$to be relatively insensitive to the pressure gradient, so this reasoning could apply. The observation that $\mathrm{N}$ decreased from lpg to hpg for sweep identification by these techniques is consistent with this idea. (Unfortunately, we no longer have the facilities to recalculate the results using the suggested value of $\mathrm{k}_{2}=\mathrm{khpg}$ so it does not prove it.) The QS algorithm is applied to the time series $\mathrm{uv}\{\mathrm{t}\}$ and we know that both $\left(\mathrm{v}^{\prime}\left\{\mathrm{y}^{+}\right\}\right)^{+}$and $\left(\overline{\mathrm{u}} \mathrm{v}\left\{\mathrm{y}^{+}\right\}\right)^{+}$are sensitive to pressure gradient (Figures $4 \mathrm{~b}$ and 5 by ME). Hence, it is not clear what to expect of the QS technique as the pressure gradient is increased.

In terms of the non-dimensional event rates $\mathrm{f}^{+}$, the sweeps show reduced rates as the favorable pressure gradient is increased (with the exception of a possible anomaly for the point near the wall) as with $\mathrm{N}$ above. This observation is consistent with those of Kline et al. and CMM. The apparent reductions in rates of sweeps are still seen but they are moderated by a reduction in $\mathrm{u} \tau^{2}$ of over forty percent from the lpg to hpg case. However, as a consequence of this reduction in $\mathrm{u}^{2}$, the non-dimensional ejection rate (from QS) then increases with pressure gradient in the outer part of the viscous layer. This result differs from the conclusions of Kline et al. and of CMM. The latter experiment applied the Vita technique to the wall shear stress signal which Eckelmann [1974] has shown to behave as the streamwise fluctuation does; thus, CMM essentially detected the equivalent of sweeps. Kline et al. counted dye streak breakups F per unit time and per unit span and noted that interpretation of their numbers is not entirely clear. For example, the shape and trajectory of a dye parcel is a consequence of its historical path from injection; though looking like ejections, a breakup observed could represent fluid pushed ahead of a sweep - or some combination.

For the opposite situation of a strong adverse pressure, Krogstad and Skare [1995] found outside the viscous layer that the frequency of sweeps increases while the frequency of ejections decreases (using 
different non-dimensionalization for time than here). Aubertine and Eaton [2005] employed quadrant splitting in the viscous layer (and across the boundary layer) to examine the effects of a mild adverse pressure gradient on the contributions to the Reynolds shear stress from the various quadrants but did not present effects on the related event rates.

In summary, whether sweep and ejection rates appear to decrease or increase with the pressure gradient depends on the quantity examined and the manner of identification (and presentation). Authors need to be cautious in their generalizations of this topic.

\subsection{Temporal Behavior of Ejections}

Some ensemble-averaged observations for the vicinity of the ejections identified by QS are shown in Figure 2. These data are from the central region of the viscous layer at $y^{+} \approx 13.2$ and 16.0 for $1 \mathrm{pg}$ and hpg, respectively. For these figures, the algorithm was selected to identify ejections by requiring $\mathrm{v}$ to be away from the wall and uv to be negative. Time of detection $\left(\mathrm{t}^{+}=0\right)$ was taken as occurrence of a maximum value of $|-\mathrm{uv}|$; consequently, the conditional average $<\mathrm{u}^{+} \mathrm{v}^{+}>$peaks at $\mathrm{t}^{+}=0$ by definition.
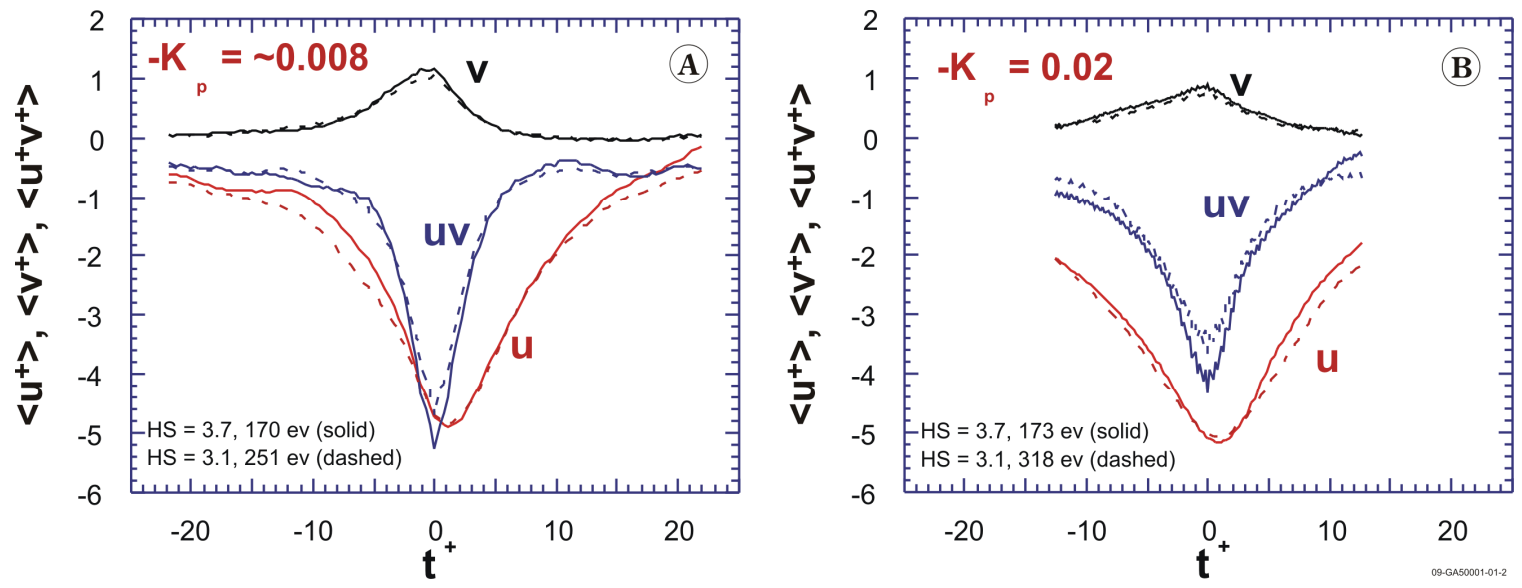

Figure 2. Transient behavior of abrupt ejections identified by the quadrant splitting technique: (a) lower pressure gradient $\left(-\mathrm{K}_{\mathrm{p}} \approx 0.008, \mathrm{y}^{+} \approx 13.2\right)$ and $(\mathrm{b})$ higher pressure gradient $\left(-\mathrm{K}_{\mathrm{p}} \approx 0.02, \mathrm{y}^{+} \approx 16\right)$.

Two high levels of threshold multiplier HS were used to identify "abrupt" ejections. One might ask the meaning of HS; essentially it sets the level of "instantaneous Reynolds shear stress" |uv| required to trigger a valid detection in multiples of (u'v'). The higher the choice of HS, the more extreme are the events required to initiate sampling (i.e., further out on the "wings" of the uv pdf) and more likely to be tripped by one of the abrupt ejections observed visually by Corino and Brodkey. One sees a slight effect on the peak value of $\left\langle\mathrm{u}^{+} \mathrm{v}^{+}>\right.$as higher thresholds lead to higher averages of the peak uv product being detected. However, the general effects on $\left\langle\mathrm{u}^{+}>\right.$and $\left\langle\mathrm{v}^{+}>\right.$behavior $\left(\right.$and $\left\langle\mathrm{u}^{+} \mathrm{v}^{+}>\right.$away from $\mathrm{t}^{+}=0$ ) are slight in this range. Comparable behavior was observed by Andreopoulos and Agui [1996] for ensemble averages based on detection of vorticity flux. In this deceleration process the maximum outward fluctuation $\left\langle\mathrm{v}^{+}>\right.$occurs slightly before the observation of $<\mathrm{uv}>\max$ in the range $-1<\mathrm{t}^{+}<0$ and it is followed by the minimum value of $<\mathrm{u}^{+}>$afterwards at $\mathrm{t}^{+}$near unity.

To examine the effects of the pressure gradient on these ensemble averages of temporal ejection behavior, we concentrate on the results with a threshold multiplier of HS $=3.7$ (solid curves) which gave about the same number of events in the two cases. The peaks are broader for the hpg case, indicating that the deceleration process is slower in non-dimensional time (correlated over a larger range of $\mathrm{t}^{+}$) or 
ejections are larger in non-dimensional space. The minimum $<\mathrm{u}^{+}>$has approximately the same value for both. Thus, the difference in minimum $\left\langle\mathrm{u}^{+} \mathrm{v}^{+}>\right.$is due to the magnitude of the outward component $\left\langle\mathrm{v}^{+}\right\rangle$. Although the threshold is the same for both lpg and hpg, the peak values differ. On average, -uv exceeded the threshold (=3.7 u'v') less for the hpg case than the lpg one, about 0.6 versus 1.6 in wall units. The corresponding peak $\left\langle\mathrm{v}^{+}>\right.$is reduced from about 1.15 to 0.85 , indicating that the most "energetic" ejections have smaller outward velocities for the higher pressure gradient. Since there is not a significant change of the peak $\left\langle\mathrm{u}^{+}>\right.$, these ejections have smaller angles with the outer flow. In the earlier paper ME likewise concluded from their probability density distributions that the vigor of ejections in the outer part of the viscous layer was less for the higher pressure gradient.

\subsection{Temporal Behavior of Sweeps as Identified via Pattern Recognition}

Background concerning the pattern recognition [WBE] and Vita techniques is presented earlier in the Introduction. Since the pattern recognition and Vita approaches both identify sweeps giving the maximum value of $\partial u / \partial y$ (which is desired for calculation of the entropy generation), it is appropriate to compare their ensemble averages. Figure 3 demonstrates these averages at $y^{+} \approx 13.2$ in our lpg channel flow. Here single point pinning was selected for the PR subroutine rather than two-point time normalization and the PR time has been converted to $\mathrm{t}^{+}$for comparison. Control parameters were adjusted to detect approximately the same number of events with both techniques $(\mathrm{AFACT}=0.4$, filter width $\mathrm{LFT}=25$, slope comparison ratio $=1.5$, ISIZE $=5$, ITPAV $=10, \mathrm{~T}_{\mathrm{avg}}{ }^{+} \approx 10.2$ and $\mathrm{k}=0.71$ ). Slight differences are seen but effectively the same patterns and information are obtained.

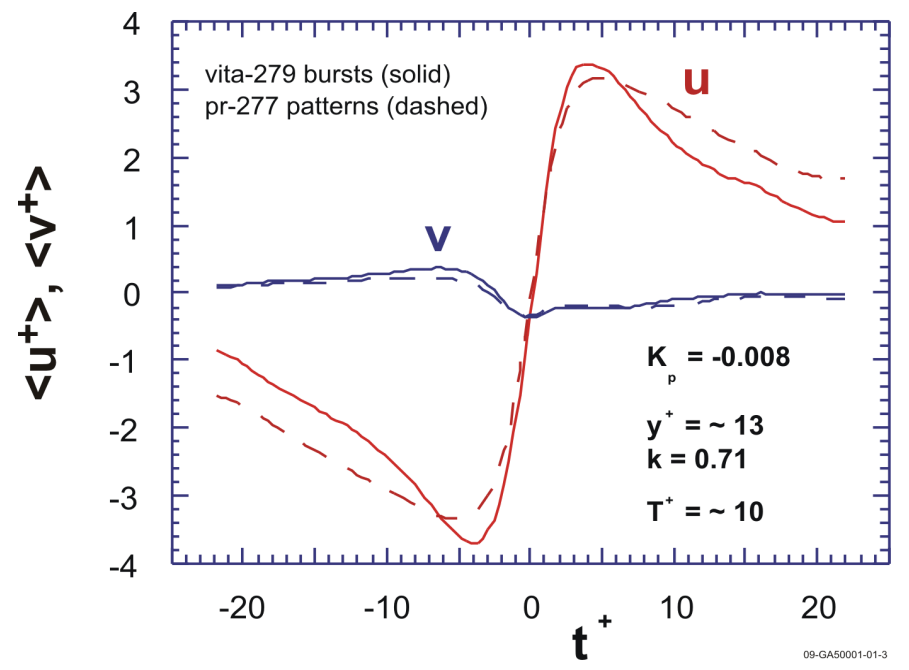

Figure 3. Comparison of transient behaviors of sweeps identified by pattern recognition technique (dashed curves) and Vita technique (solid curves) at $\mathrm{y}^{+} \approx 13.2$ in viscous layer of the lower pressure gradient experiment.

The ensemble averages for Vita have slightly higher extreme values. For $\mathrm{t}^{+}<4$, deceleration of $<\mathrm{u}^{+}>$ is observed and $<\mathrm{v}^{+}>$is outward; an ejection is occurring. For PR the maximum outward velocity occurs at $\mathrm{t}^{+} \approx-9.9$ ahead of the minimum in $<\mathrm{u}^{+}>$at $\mathrm{t}^{+} \approx-5.1$ (with Vita detection the corresponding times are $\mathrm{t}^{+} \approx-6.6$ and -4.0 ). These portions of the averages correspond to those shown in Figure 2 from ejection detection except the relative timing of peaks differs somewhat. The wall-normal fluctuation $<_{\mathrm{v}}^{+}>$ 
becomes inward at $\mathrm{t}^{+} \approx-2.9$ as the $<\mathrm{u}^{+}>$deceleration begins to abate. This section of the average from $\mathrm{t}^{+} \approx-2.9$ to about +0.1 corresponds to a wallward interaction. Then the right side of the Figure 3 beyond $\mathrm{t}^{+} \approx 0.1$ shows the characteristic behavior of a sweep.

Wallace, Brodkey and Eckelmann compared their deduced event durations to those of Blackwelder and Kaplan. With a slope comparison ratio of unity in the PR technique, they found average nondimensional pattern lengths $\mathrm{T}^{*}\left(=2 \mathrm{TU}_{\mathrm{c}} / \mathrm{s}\right.$ where $\mathrm{T}$ is duration $)$ of about 3.3 and 2.6 at $\mathrm{y}^{+}=15$ and 30 , respectively, for accepted patterns. Blackwelder and Kaplan found that the bursting sequence they detected at $\mathrm{y}^{+}=15$ had $\mathrm{TU}_{\infty} / \delta \approx 2.8$ for an average duration ( $\delta$ is the boundary layer thickness).

Eckelmann and Wallace [1981] compared the two techniques from several other viewpoints, using signals from a five-sensor probe for PR. From their gradient measurements, they observed that in the wall-normal direction the $<\mathrm{u}>$ pattern obtained at $\mathrm{y}+\Delta \mathrm{y}$ is very similar to the pattern obtained at $\mathrm{y}$ with only a phase difference; in the spanwise direction reduction of amplitude occurs but no phase shift. These observations are consistent with those from Vita by Blackwelder and Kaplan using rakes oriented in the wall-normal and spanwise directions. One difference noted was that, with the control parameters they used, the maximum ensemble-average $<$ uv $>$ was greater for Vita than with PR, indicating that the Vita technique is a sharper method for detecting Reynolds-shear-stress-producing structures.

As also shown earlier by WBE, Eckelmann and Wallace [1981] and others, Figure 3 demonstrates that the observed temporal behavior of sweeps is essentially the same whether identified by PR or Vita. Since the present study is interested in the simultaneous temporal behavior ( of $\mathrm{S}_{\mathrm{t}, \mathrm{uy}}{ }^{+}$) at different wall distances, we conduct our further examinations using the Vita technique since our implementation of it provides the desired ensemble averages directly as functions of $\mathrm{t}^{+}$.

\subsection{Effects of Pressure Gradient on Temporal Behavior of Sweeps}

A general overview of the sweep passage is presented as Figure 4 for the two pressure gradients with three probe locations each. All results include 300 or more detected events (called "bursts" in the processing code). The X-probe and wall sensor provide four ensemble-averages: $\left\langle\mathrm{u}>^{+},\left\langle\mathrm{v}>^{+},\left\langle\mathrm{uv}>^{+}\right.\right.\right.$and $<\tau_{\mathrm{W}}{ }^{+}$. For the hpg run, locations are $\mathrm{y}^{+} \approx 7.4,16.0$ and 26.4 while they are 5.6, 13.2 and 22.3 for the $\operatorname{lpg}$ experiment. The wall shear stress trace is multiplied by a constant for visibility; otherwise the same scaling is applied to all subfigures for easy comparison. The timing of some of the key events is included in Table 1. Due to so-called "jitter" ( $\sim$ random phase differences between key events) the amplitudes of the ensemble averages are moderated at large times $\left|\mathrm{t}^{+}\right|$. Therefore, at large positive or negative times, the observations might be considered to be qualitative whereas near $\mathrm{t}^{+}=0$ they are expected to be reasonably quantitative.

Since measurement of $\tau_{\mathrm{W}}\{\mathrm{t}\}$ is common to all probe locations and Eckelmann [1974] has shown that the instantaneous wall shear stress is similar to the instantaneous $\mathrm{u}\{\mathrm{t}\}$ fluctuation, the non-dimensional time $\mathrm{t}_{\mathrm{W}}{ }^{+}$is based on equivalent Vita identification at the wall sensor. As indicated by Eckelmann and Wallace [1981], the Vita detection with the u-signal typically occurs at the maximum positive gradient of $<\partial \mathrm{u} / \partial \mathrm{t}>$; therefore, the equivalent detection time $\mathrm{t}_{\mathrm{W}}{ }^{+}=0$ is defined as the time of the maximum positive gradient of $\left\langle\partial \tau_{\mathrm{W}} / \partial \mathrm{t}>\right.$. Thus, the traces for the three locations are placed on a simultaneous time basis as they would be if measured with a rake. These temporal distributions can be qualitatively considered to be "reverse images" of the spatial distributions in the vicinity of the average sweep front (i.e., $\mathrm{t}^{+}$corresponds to $-\mathrm{x}^{+}$). 

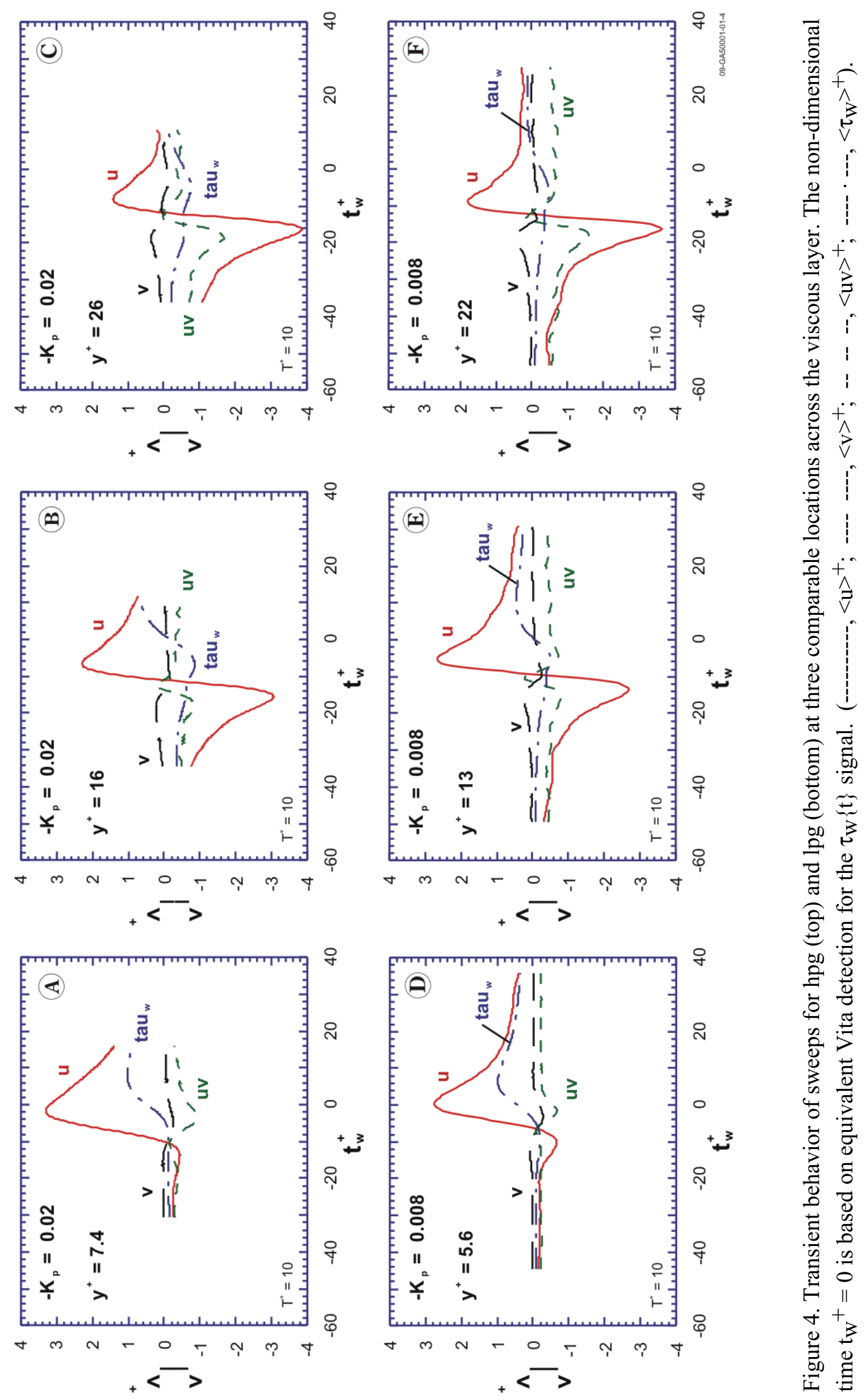
Table 1. Non-dimensional times of occurrences of interesting events in the Vita signatures (all times in terms of $\mathrm{t}_{\mathrm{W}}{ }^{+} ; \mathrm{t}_{\mathrm{W}}{ }^{+}=0$ at equivalent wall detection).

\begin{tabular}{|c|c|c|c|}
\hline$-\mathrm{K}_{\mathrm{p}} \approx 0.02, \mathrm{hpg}$ & $y+\approx 7.4$ & $y+\approx 16.0$ & $y+\approx 26.4$ \\
\hline $\operatorname{Max} v+$ at $t+$ & $+0.037 @-15.5$ & $+0.235 @-18.0$ & $+0.364 @-18.8$ \\
\hline Min $u v+$ at $t+$ & $-0.390 @-19.5$ & $-0.833 @-17.9$ & $-1.69 @-18.4$ \\
\hline Min Suy+ at $t+$ & $0.368 @-15.5$ & $0.062 @-16.2$ & $\begin{array}{l}0.0019 @-21.4 \text { and } \\
0 @-10.1,+1.5 \text { and } \\
+7.7\end{array}$ \\
\hline Min $u+$ at $t+$ & $-0.435 @-13.6$ & $-3.05 @-15.5$ & $-3.87 @-16.2$ \\
\hline$t w+$ for $v+=0$ & -12.2 & -14.2 & $\begin{array}{l}-14.4,-12.2, \quad-7.4 \\
\text { (oscillation) }\end{array}$ \\
\hline Max uv+ at $t+$ & $-0.157 @-10.7$ & $+0.256 @-12.5$ & $+0.0344 @-13.1$ \\
\hline Min $v+$ at $t+$ & $-0.266 @-5.0$ & $-0.199 @-11.8$ & $\begin{array}{l}-0.043 @-13.1 \text { and } \\
-0.115 @-3.7\end{array}$ \\
\hline "Front" detection & -7.3 & -11.4 & -12.65 \\
\hline Max Suy+ at $t+$ & $0.891 @-4.8$ & $0.275 @-9.6$ & $\begin{array}{l}0.053 @-12.5 \text { and } \\
0.023 @-7.4\end{array}$ \\
\hline $\operatorname{Max} u+$ at $t+$ & $+3.31 @-1.8$ & $+2.31 @-6.6$ & $+1.41 @-8.1$ \\
\hline Min uv+ at $t+$ & $-0.911 @-3.5$ & $\begin{array}{l}-0.332 @-5.5 \text { and } \\
-0.483 @+10.3 \text { and } \\
11.0\end{array}$ & $\begin{array}{l}-0.505 @-5.6 \text { and } \\
-0.536 @+8.1\end{array}$ \\
\hline$-\mathrm{K}_{\mathrm{p}} \approx 0.02, \mathrm{hpg}$ & Interp. $y+\approx 5.6$ & Interp. $y+\approx 13.2$ & Interp. $\mathrm{y}^{+} \approx 22.3$ \\
\hline Min Suy+ at $t+$ & $0.531 @-15.5$ & $0.0852 @-15.8$ & $\begin{array}{l}0.0192 @-18.1 \text { and } \\
0.0083 @-6.3\end{array}$ \\
\hline Min $u+$ at $t+$ & $-0.252 @-12.2$ & $-2.38 @-15.5$ & $-3.85 @-15.8$ \\
\hline Max Suy+ at $t+$ & $1.30 @-1.8$ & $0.475 @-8.8$ & $0.0914 @-12.2$ \\
\hline $\operatorname{Max} u+$ at $t+$ & $+2.92 @-1.5$ & $+2.29 @-6.3$ & $+2.01 @-7.4$ \\
\hline$-\mathrm{K}_{\mathrm{p}} \approx 0.008, \quad \mathrm{lpg}$ & $y+\approx 5.6$ & $\mathrm{y}+\approx 13.2$ & $y+\approx 22.3$ \\
\hline $\operatorname{Max} v+$ at $t+$ & +0.0814@-13.2 & $+0.249 @-16.2$ & $+0.385 @-19.2$ \\
\hline Min Suy+ at $t+$ & $0.442 @-13.3$ & $0.0848 @-16.2$ & $\begin{array}{l}0.00054 @-17.7 \text { and } \\
0 @-7.7 \text { and }-0.5\end{array}$ \\
\hline Min uv+ at $t+$ & $-0.241 @-16.5$ & $-0.801 @-15.1$ & $-1.60 @-18.0$ \\
\hline Min $u+$ at $t+$ & $-0.653 @-10.3$ & $-2.68 @-13.3$ & $-3.61 @-16.2$ \\
\hline$t w+$ for $v+=0$ & -8.4 & -12.0 & -14.7 \\
\hline Max $u v+$ at $t+$ & $-0.888 @-7.0$ & $+0.230 @-10.4$ & $+0.194 @-13.6$ \\
\hline Min $v+$ at $t+$ & $-0.279 @-3.3$ & $-0.267 @-9.3$ & $\begin{array}{l}-0.141 @-13.3 \text { and } \\
-0.161 @-6.3\end{array}$ \\
\hline "Sweep" detection & -4.42 & -9.3 & -12.89 \\
\hline Max Suy+ at $t+$ & $1.149 @-1.1$ & $0.433 @-7.7$ & $\begin{array}{l}0.640 @-11.4 \text { and } \\
0.067 @-5.5\end{array}$ \\
\hline $\operatorname{Max} u+$ at $t+$ & $+2.78 @+0.3$ & $+2.67 @-4.9$ & $+1.79 @-8.8$ \\
\hline Min uv+ at $t+$ & $-0.671 @-1.5$ & $-0.729 @-4.2$ & $\begin{array}{l}-0.622 @-6.6 \text { and } \\
-0.677 @-2.2\end{array}$ \\
\hline
\end{tabular}


The results from the hpg experiment appear to be approximately the same as for the well-studied lpg at corresponding locations. For both situations, the ensemble averages generally show first a Q2 deceleration continuing briefly $\left(\Delta \mathrm{t}^{+} \approx 2-4\right)$ as a Q3 wallward interaction as the Q4 sweep interface approaches. Evidence of sweep front "arrival" can be considered to be observation of an increase in $<\mathrm{u}^{+}$ from its minimum and $<\mathrm{v}>^{+}$becoming negative. Here interface passage then is defined as the time when $<\mathrm{u}>^{+}$crosses zero. The "instantaneous Reynolds shear stress" $<\mathrm{uv}>^{+}$shows very little positive contribution in the vicinity of the front passage and none at the near-wall probe position. Near the wall the deceleration phase of $\left\langle\mathrm{u}^{+}\right.$is slight while during acceleration it reaches values near three. This observation is another indication that sweeps dominate the Reynolds stress near the wall; it is also evident in the $<\mathrm{uv}^{+}{ }^{+}$variation which has its minimum in this phase. Also after front passage here $<-\mathrm{uv}>^{+}$is much greater that the mean Reynolds shear stress. Away from the wall the deceleration is much greater, peaking at values near -4 while the acceleration does not exceed $\left\langle\mathrm{u}>^{+} \approx 2\right.$. The outward component $\langle\mathrm{v}\rangle^{+}$is likewise greater away from the wall during its ejection phase, corresponding to earlier observations that ejections dominate the Reynolds shear stress there (as demonstrated by the $<\mathrm{uv}^{+}{ }^{+}$signature as well). In the central region of the viscous layer the transient behaviors are intermediate in magnitude relative to those near and away from the wall; the $<\mathrm{u}>^{+}$and $<\mathrm{v}>^{+}$distributions are near anti-symmetric, with the minima approaching the same magnitudes as the maxima. As the wall is approached the durations of the phases become broader corresponding to slower velocities.

While examination of transient turbulent production is not an aim of the present study, some basic comments may be made in passing. The following observations apply to both hpg and lpg cases. If one defines an "instantaneous production" of tke as $<-\mathrm{uv}>^{+}\left(\partial \mathrm{U}^{+} / \partial \mathrm{y}^{+}\right)$as by Diorio, Kelley and Wallace [2007], one sees that the time $\mathrm{t}_{\mathrm{W}}{ }^{+}$of its maximum varies with distance from the wall. (From their PR measurements, Eckelmann et al. [1977] showed that - at $\mathrm{y}^{+} \approx 30$ for a lpg experiment - their instantaneous production was significantly greater than that defined by Diorio, Kelley and Wallace but their peak values occurred at approximately the same "normalized time.") Since $\left(\partial \mathrm{U}^{+} / \partial \mathrm{y}^{+}\right)$is independent of $\mathrm{t}^{+}$at any $\mathrm{y}^{+}$, one can examine the behavior of this version of "instantaneous production" by observation of the signatures for "instantaneous Reynolds shear stress," <-uv $>^{+}$. Wilhelm, Härtel and Eckelmann [1998] found that an "instantaneous Reynolds shear stress" (-puv) generally showed high values and, therefore, intense production after interface passage. For the present data, near the wall the maximum $<-$ uv $>^{+}$would likewise occur after the front passage. At this position Figure $10 \mathrm{~b}$ of WHE actually shows a slight maximum of $\langle-\mathrm{uv}\rangle^{+}$a bit ahead of their interface (with detection at $\mathrm{y}^{+} \approx 12.3$ ) but their front may be more curved than ours there. In the region of greatest mean production of tke, i.e., in the central region of the viscous layer for lpg, local maxima of "instantaneous production" would occur both before and after the frontal passage; for hpg the same situation appears but the peak value is greater ahead of the front. In the outer part of the viscous layer, this maximum "instantaneous production" would be predominantly earlier than the frontal passage, during the ejection phase. (However, WHE did not present any significant values of $\left\langle-\mathrm{uv}>^{+}\right.$in the ejection phase ahead of their front so comparison with the present data cannot be made there.)

The ensemble averages for both hpg and lpg are approximately the same at the same nominal locations $\mathrm{y}^{+}$. One may conclude that increasing the favorable pressure gradient does not cause large effects on the sweep behavior. CMM had about the same comment concerning ensemble averages of $\tau_{\mathrm{W}}\{\mathrm{t}\}$ until $\mathrm{K}_{\mathrm{V}}$ becomes large. However, there are differences in details. 
In comparison to the lpg experiment, near the wall for the higher pressure gradient there is a broader, higher peak in $\left\langle\mathrm{u}^{+}{ }^{+}\right.$with less deceleration in advance, $\left\langle\mathrm{v}>^{+} \text {outward is less and }<\text {-uv }\right\rangle^{+}$is greater in the sweep phase. Here there is greater lag between the $\mathrm{u}$ and $\tau_{\mathrm{W}}$ detections. In the central region ones sees $a$ smaller contribution of $<-\mathrm{uv}\rangle^{+}$to Reynolds shear stress from inflow after the sweep passage for hpg than for lpg results (whereas ahead of the detection there, the outflow provides approximately the same peak values of $<-\mathrm{uv}\rangle^{+}$from both experiments). Further from the wall $\left.<\mathrm{v}\right\rangle^{+}$is not as negative after the interface passage. So the sweep flow angle towards the wall is less there. And at these locations $<-$ uv $>^{+}$is slightly less for hpg in the sweep phase than for lpg. In the outer viscous layer the (non-dimensional) lag in detection is almost the same for both pressure gradients. In general, the ensemble averages for the hpg experiment are broader or slower in non-dimensional time and longer in non-dimensional space than the lpg results. The "peak-to-peak" differences in $\left\langle\mathrm{v}\left\{\mathrm{t}^{+}\right\}>^{+}\right.$are less for the hpg case.

For both hpg and lpg conditions, one sees that the time interval between the u-detection and the $\tau_{\mathrm{W}^{-}}$ detection (i.e., their respective maximum gradients) increases with distance from the wall. This result corresponds to the observations of Kreplin and Eckelmann [1979b] considering fronts. The fronts are pictured as being angled from the wall so the flow structure will intersect a probe away from the wall earlier than one near the wall. With DNS Wilhelm, Härtel and Eckelmann [1998] showed further evidence that the fronts first discussed by Kreplin and Eckelmann [1979b] are essentially identical to extended shear layers in near-wall flow. In good agreement with the experimental results, the front was found to be inclined to the wall from the linear layer into the logarithmic region. In the present results, there is an apparent change in the shape of the fronts as the pressure gradient is increased. To consider differences in front shapes, one can compare the non-dimensional lag from the u-detection to the $\tau_{\mathrm{W}}$-detection relative to $\mathrm{y}^{+}$for the two pressure gradients. Figure 5 provides this comparison as the difference $\left(\mathrm{t}_{\mathrm{W}}{ }^{+}-\mathrm{t}_{\mathrm{u}}{ }^{+}\right)$where $\mathrm{t}_{\mathrm{u}}{ }^{+}$is the non-dimensional time relative to the u-detection. For the lpg experiment, the points near the wall are almost linear relative to the origin and then there is greater curvature to the outer point. The hpg front is more rounded than for lpg, appearing near parabolic and having a more acute slope near the wall. Propagation of the hpg front in the near wall region is sloweror one might interpret the figure as showing that there is quicker propagation in the outer region of the hpg viscous layer giving a flatter form there.

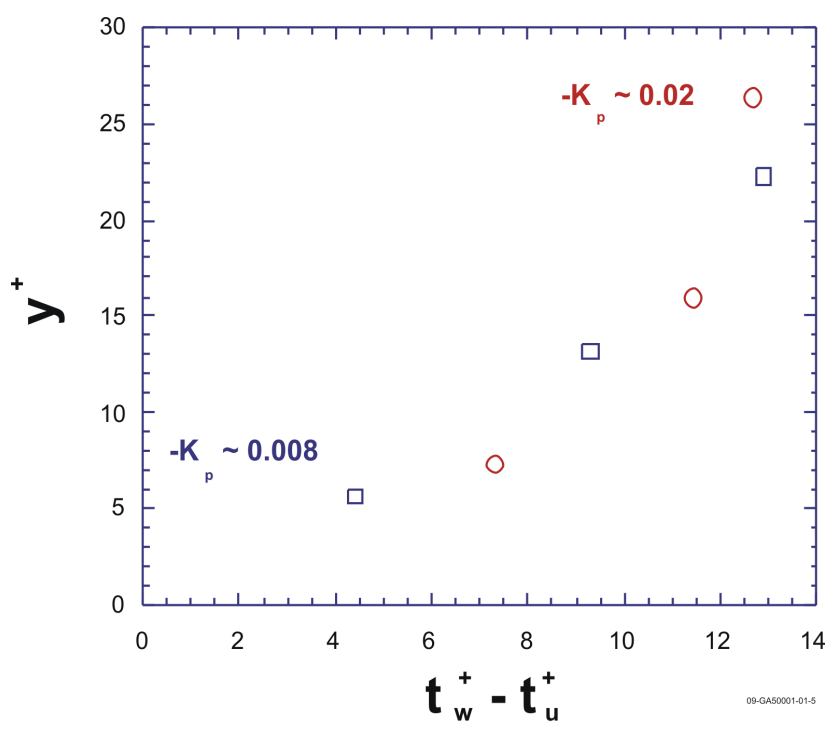

Figure 5. Front shape as indicated by non-dimensional time lag between u-detection and $\tau_{\mathrm{W}}$-detection (circles, $-\mathrm{K}_{\mathrm{p}} \approx 0.02$; squares, $-\mathrm{K}_{\mathrm{p}} \approx 0.008$ ). 
Since the X-probe locations are not exactly the same in distance $\mathrm{y}^{+}$for the two pressure gradients, one may wonder whether some differences in detail are due to differences in non-dimensional position. Our conclusions above for front timing are valid since Figure 5 is plotted in terms of actual nondimensional probe locations. To compare the ensemble averages at equal distances, values of $<\mathrm{u}^{+}$for the hpg experiment were interpolated to the same positions as in the lpg measurements. Quadratic interpolation of $<\mathrm{u}^{+}\left\{\mathrm{t}_{\mathrm{W}}{ }^{+}\right\}>$was applied to determine values of $\mathrm{u}^{+}$at the same non-dimensional instants $\mathrm{t}_{\mathrm{W}}{ }^{+}$at each of the locations $\mathrm{y}^{+}$. Then quadratic functions were fitted in the wall normal direction to deduce $<\mathrm{u}^{+}\left\{\mathrm{y}^{+}, \mathrm{t}_{\mathrm{W}}{ }^{+}\right\}>$at each instant at each probe location in the viscous layer. The results of the comparisons of shape and magnitudes at the same values of $\mathrm{y}^{+}$(not shown) for the two pressure gradients are essentially the same as the observations presented relative to Figure 4 . The lag between $u-$ and $\tau_{\mathrm{W}}$-detection is consistent with Figure 5. When compared at the same $\mathrm{y}^{+}$the interpolated values of $<\mathrm{u}^{+}>$ for the hpg conditions show slightly less deceleration ahead of the u-detection than at the actual probe locations further from the wall. The general trends observed for $\left\langle\mathrm{u}^{+}>\right.$are still valid.

In summary, the main differences observable between the two pressure gradients during sweep passage are (1) changes in the time lag between detections - representing modification of the shape of the sweep front and the sweep angle with the wall, (2) modification of the magnitude of $\left\langle\right.$ uv $>^{+}$with wall distance and (3) broadening of the sweep fronts and ejections for the hpg experiment.

\subsection{Temporal Behavior of Entropy Generation Rates and Effects of Pressure Gradient}

As the objective of the present work involves examining the transient entropy generation rates and since its maximum value occurs during the sweep, identification of the sweep and determination of the behavior in its vicinity are needed. Application of the Vita technique to the time series $u\{t\}$ provides the means as it is known to detect maximum $\partial \mathrm{u} / \partial \mathrm{t}$ and, correspondingly, maximum $\partial \mathrm{u} / \partial \mathrm{y}$ (hence maximum $\mathrm{S}$ t,uy).

Our "synchronized" values of $<\mathrm{u}^{+}\left\{\mathrm{t}_{\mathrm{W}}{ }^{+}\right\}>$and $<\mathrm{v}^{+}\left\{\mathrm{t}_{\mathrm{W}}{ }^{+}\right\}>$allow calculation of a few terms contributing to $<\mathrm{S}_{\mathrm{t}}{ }^{+}>$including the dominant one. (Unfortunately, our data do not include spanwise measurements to deduce $\left(\tilde{\partial \mathrm{u}^{+}} / \partial \mathrm{z}^{+}\right)^{2}$ which is expected to be second in importance.) Quadratic interpolation of $<\mathrm{u}^{+}\left\{\mathrm{t}_{\mathrm{W}}{ }^{+}\right\}>$and $<\mathrm{v}^{+}\left\{\mathrm{t}_{\mathrm{W}}{ }^{+}\right\}>$was applied to determine values of these velocity components at the same non-dimensional instants $\mathrm{t}_{\mathrm{W}}{ }^{+}$in each of the experimental runs. After adding the mean values to obtain the instantaneous quantities at these instants, quadratic functions were fitted in the

wall normal direction to deduce the dominant contribution $\mathrm{S}_{\mathrm{t}, \mathrm{uy}}{ }^{+}=\left(\partial \tilde{\mathrm{u}}{ }^{+} / \partial \mathrm{y}^{+}\right)^{2}$ at each probe location in the viscous layer. Thus, this process provides deduced synchronized ensemble-average results for $S_{t, u y}{ }^{+}$ as functions of $\mathrm{t}_{\mathrm{W}}{ }^{+}$for each probe location in the viscous layer.

From Figure 4 by Antonia, Kim and Browne [1991] one can estimate values of the mean square gradients which could be deduced for evaluation of $\mathrm{S}^{+}$from X-probe data perpendicular to wall. In the viscous layer, the time-mean values of $\left(\partial \mathrm{u}^{+} / \partial \mathrm{x}^{+}\right)^{2},\left(\partial \mathrm{v}^{+} / \partial \mathrm{y}^{+}\right)^{2}$ and $\left(\partial \mathrm{v}^{+} / \partial \mathrm{x}^{+}\right)^{2}$ are predicted to be 0.0002 to $0.002,0.001$ to 0.003 and 0 to 0.001 , respectively. These values are small compared to unity, the order of the direct dissipation $\left(\partial \mathrm{U}^{+} / \partial \mathrm{y}^{+}\right)^{2}$ near the wall. In the ensemble average $<\mathrm{u}^{+}\left\{\mathrm{t}_{\mathrm{W}}{ }^{+}\right\}>$the 
maximum value of $\partial \mathrm{u}^{+} / \partial \mathrm{t}^{+}$is about 1.5 and occurs at the point of $\mathrm{u}$-detection (the run at $\mathrm{y}^{+} \approx 22.2$ and $-\mathrm{K}_{\mathrm{p}} \approx 0.008$ shows the steepest slope). With a conservative choice of $\mathrm{V}_{\mathrm{c}}^{+} \approx 11$ for the convection velocity from Wilhelm, Härtel and Eckelmann [1998], one may estimate the maximum "instantaneous" value of $<\partial \tilde{\mathrm{u}}^{+} / \partial \mathrm{x}^{+}>2$ to be about 0.02 - which is greater than the predicted mean value of $\mathrm{S}_{\mathrm{t}}{ }^{+}$at this location but small relative to the desired peak values of $\mathrm{S}_{\mathrm{t}, \mathrm{uy}}{ }^{+}$. The estimated maximum values of $<\partial \mathrm{v}^{+} / \partial \mathrm{y}^{+}>2$ and $<\partial \mathrm{v}^{+} / \partial \mathrm{x}^{+}>2$ are found to be small in the same sense.

The new key results are provided in Figures $6 \mathrm{a}$ and $\mathrm{b}$. To our knowledge, this temporal behavior has not even been deduced for lpg previously. Figure $6 \mathrm{a}$ provides $<\mathrm{S}_{\mathrm{t}}, \mathrm{uy}{ }^{+}>$for the $\mathrm{lpg}$ experiment as dashed lines and Figure $6 \mathrm{~b}$ shows this ensemble average for the hpg one as solid curves. As before, the time $\mathrm{t}_{\mathrm{W}}{ }^{+}=0$ is equivalent to detection of interface passage based on the signal of the wall sensor which is common for each run. Also shown as horizontal dotted lines on subfigure 6a are the time-mean values of $\left(\mathrm{S}_{\mathrm{t}}\right)^{+}$for the low pressure gradient experiment, including contributions from all spatial gradients (deduced from the DNS of Spalart for zpg [1988; McEligot et al., 2008b]).
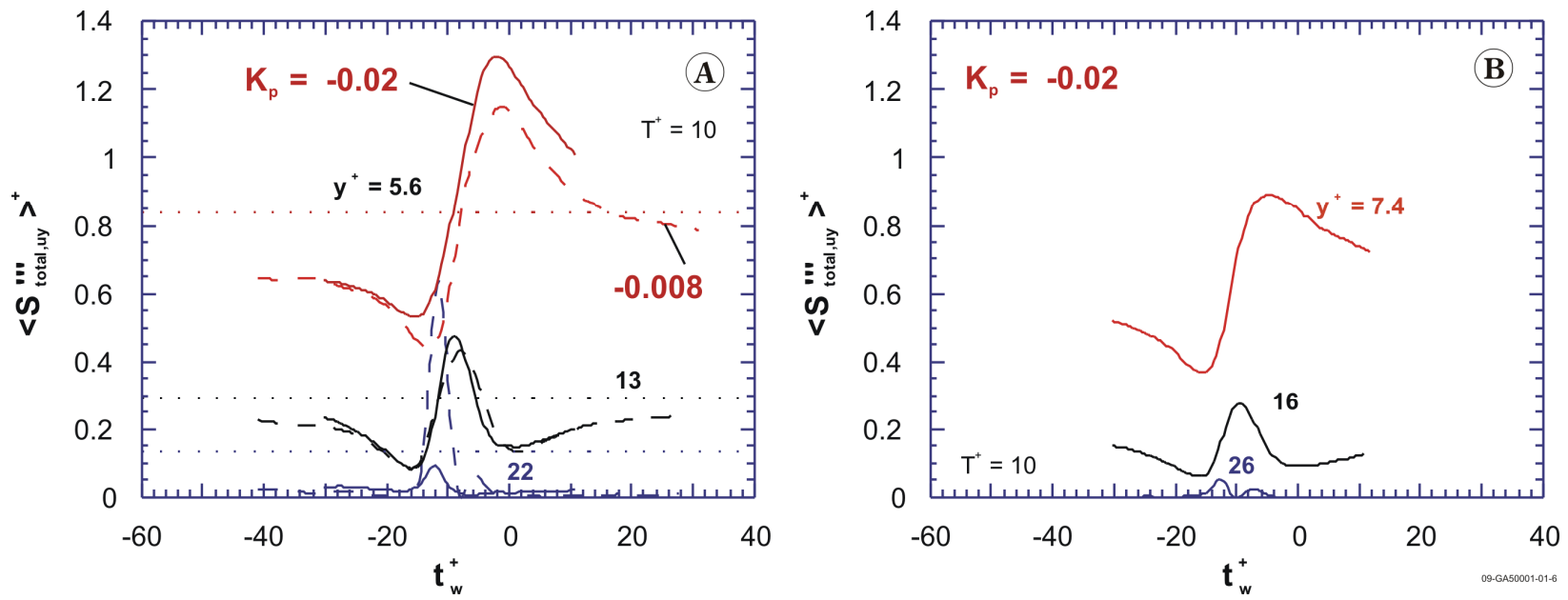

Figure 6. Transient behavior of the dominant contribution to pointwise total entropy generation rate; high pressure gradient case $\left(-\mathrm{K}_{\mathrm{p}} \approx 0.02\right)$ identified with solid curves and low pressure gradient $\left(-\mathrm{K}_{\mathrm{p}} \approx 0.008\right)$ with dashed curves: (a) at probe locations for lpg experiment and (b) for hpg experiment.

One sees that the general trends with $\mathrm{y}^{+}$and $\mathrm{t}_{\mathrm{W}}{ }^{+}$are the same in the lpg experiment and the hpg one but with some differences, particularly for the values nearer to the outer edge of the viscous layer. The levels of entropy generation rate decrease as $\mathrm{y}^{+}$increases; by the edge of the viscous layer $\left(\right.$say $\mathrm{y}^{+}>20$ or 30 ), $<\mathrm{S}_{\mathrm{t}, \mathrm{uy}}{ }^{+}>$is small compared to the mean value of $\mathrm{S}_{\mathrm{t}}{ }^{+}$at the wall (predicted to be about 1.1 to 1.2). This transverse variation is consistent with DNS predictions of the time-mean entropy generation rate [McEligot et al., 2008a,b]. Also the breadths of the peaks increase approaching the wall, indicating that the high temporal values of entropy generation rates last for longer durations near the wall. The main increase in $\mathrm{S}_{\mathrm{t}, \mathrm{uy}}{ }^{+}$at a probe location $\mathrm{y}^{+}$appears near the front passage indicated by the earlier ensemble averages of $<\mathrm{u}>^{+}$.

The $<\mathrm{S}_{\mathrm{t}, \mathrm{uy}}{ }^{+}>$results nearer the edge of the boundary layer show multiple peaks. Examination of these results with a logarithmic scale for the ordinate (not included) shows steep regions and very low 
values between the peaks, indicating that $<\mathrm{S}_{\mathrm{t}, \mathrm{uy}}{ }^{+}>$may be approaching zero at these locations. From the tabulated values one can see that at some instants the velocity gradient $\partial \tilde{u}^{+} / \partial y^{+}$changes from positive to negative and vice versa. These observations are consistent with the measurements of Eckelmann and Randolph [1993, their Fig. 1] using a gradient probe; they found that for $\mathrm{y}^{+}$greater than about ten, some individual samples of $\partial \tilde{\mathrm{u}}^{+} / \partial \mathrm{y}^{+}$were negative. This effect was not seen in our ensembles for $\mathrm{y}^{+}$of sixteen and less where averages of more than 300 events were taken, thereby moderating effects of any extreme events. For the outer traces $\left(\mathrm{y}^{+} \approx 22.3\right.$ and 26.4) the highest peaks come from positive values of $\left(\partial \tilde{u}^{+} / \partial y^{+}\right)$during sweep passage but they are followed by secondary peaks which correspond to negative gradients during the following deceleration phase.

Brodkey et el. [1974] note that total dissipation (and therefore instantaneous total entropy generation rate) can be considered as a composite of local processes, one of which (observed visually) occurs in the high velocity gradient region that separates the decelerated flow (from which ejections originate) from the higher-speed sweep. If one interprets the detection as the instant of passage of an ejection/sweep interface, one can see that the peak of $<\mathrm{S}_{\mathrm{t}, \mathrm{uy}}{ }^{+}>$generally is near the $<\mathrm{u}^{+}\left\{\mathrm{t}_{\mathrm{W}}{ }^{+}\right\}>$maximum and therefore - occurs slightly after the front passage. This delay is greater near the wall than for the measurements near the edge of the viscous layer. These observations are consistent with others for lpg in showing that the maximum spatial gradient occurs near the front [Eckelmann and Wallace, 1981; Randolph, Eckelmann and Nychas, 1987]. From their DNS and application of Visa sampling, WHE also essentially show that the highest values of $\left\langle\mathrm{St}_{\mathrm{t}, \mathrm{uy}}{ }^{+}>\right.$should occur shortly after the interface passage.

Further insight is obtained by comparing these figures to the related measurements of the fluctuations shown earlier in Figures 4 (and to our tabulated results). Typical ensemble averages for these apparent events, show a decrease in $\left\langle\mathrm{S}_{\mathrm{t}, \mathrm{uy}}{ }^{+}>\right.$corresponding to the deceleration in $\left\langle\mathrm{u}^{+}>\right.$as $\mathrm{t}_{\mathrm{W}}{ }^{+}$increases, then a rapid increase with the front passage, followed by a gradual recovery to/towards previous levels. Though the temporal behavior of $<\mathrm{S}_{\mathrm{t}, \mathrm{uy}}{ }^{+}>$is deduced from the fluctuation $\left(\mathrm{u}\left\{\mathrm{t}_{\mathrm{W}}{ }^{+}\right\}\right)^{+}$and the mean velocity $\mathrm{U}^{+}\left\{\mathrm{y}^{+}\right\},<\mathrm{S}$, uy ${ }^{+}>$is not an image of $<\mathrm{u}\left\{\mathrm{t}^{+}\right\}>^{+}$. Near the wall the resemblance is close except that before sweep passage the reduction of $\left\langle\mathrm{St}_{\mathrm{t}, \mathrm{uy}}>^{+}\right.$is greater than for $\left\langle\mathrm{u}\left\{\mathrm{t}^{+}\right\}>^{+}\right.$; this region is where sweeps dominate the Reynolds shear stress. Near the outer edge of the viscous layer, where ejections are more important for $\overline{\mathrm{u}} \overline{\mathrm{v}}$ than sweeps are, the instantaneous positive and negative gradients of $\partial \mathrm{u}^{+} / \partial \mathrm{y}^{+}$lead to multiple oscillations of $<\mathrm{S}_{\mathrm{t}, \mathrm{uy}}{ }^{+}>$as opposed to the single oscillation of $<\mathrm{u}\left\{\mathrm{t}^{+}\right\}>^{+}$.

The lowest entropy generation rate occurs during the Q2 deceleration phase and its maximum is slightly after interface passage. In Section 3.4 details of ensemble averages of the "instantaneous production" of tke (which later generates entropy by dissipating), as defined by Diorio, Kelley and Wallace [2007], have been treated. At the point near the wall this maximum production rate appears after sweep front passage, at about the same time as the maximum entropy generation rate. At the other two points further out, the largest production rate appears earlier than sweep interface passage while the maximum $<\mathrm{S}_{\mathrm{t}, \mathrm{uy}}{ }^{+}>$still occurs during the sweep slightly after the interface passage.

Times of the occurrences of the maximum and minimum $<\mathrm{S}_{\mathrm{t}, \mathrm{uy}}{ }^{+}>$and some other pertinent features are further documented in Table 1. As the front approaches, first events are minimum $<\mathrm{S}_{\mathrm{t}, \mathrm{uy}}{ }^{+}>$, maximum $<\mathrm{v}^{+}>$and minimum $<\mathrm{uv}^{+}{ }^{+}$with their apparent sequences differing with wall distance and sometimes with pressure gradient. In several cases the maximum outward $<\mathrm{v}^{+}>$, presumably from 
ejections, appears at approximately the same time as the minimum $<\mathrm{S}_{\mathrm{t}, \mathrm{uy}}{ }^{+}>$; near the wall the minimum $<\mathrm{uv}>^{+}$appears before these other two, during the gradual deceleration and outflow. Next comes the minimum $<\mathrm{u}^{+}>$or maximum deceleration, usually followed by the maximum $<\mathrm{uv}>^{+}$or minimum "instantaneous Reynolds shear stress" and then detection of the front. The minimum "instantaneous Reynolds shear stress" - from the wallward interaction ahead of the front - occurs sequentially later as the wall is approached, corresponding to the slope of the front itself. Shortly after interface passage the maximum $<\mathrm{St}_{\mathrm{t}, \mathrm{uy}}{ }^{+}>$occurs followed, in turn, by maximum $<\mathrm{u}^{+}>$. The time interval between the first of these events (either the maximum outward $<\mathrm{v}^{+}>$or the maximum "instantaneous Reynolds shear stress") and the final event, maximum $<\mathrm{u}^{+}>$from the sweep, is largest near the wall.

As would be expected from examination of $<\mathrm{u}^{+}>$, one sees the same trends with respect to $\mathrm{t}^{+}$and $\mathrm{y}^{+}$ at the two different pressure gradients but some differences are evident. Comparison of the deduced $<\mathrm{S}_{\mathrm{t}, \text { uy }}{ }^{+}>$results for $-\mathrm{K}_{\mathrm{p}} \approx 0.02$ (Figure $6 \mathrm{~b}$ ) with those for $-\mathrm{K}_{\mathrm{p}} \approx 0.008$ (Figure 6a, dashed curves) for comparable nominal probe positions could lead one to conclude that the effect of a strong pressure gradient is to reduce this temporal component of entropy generation, both its levels and its maxima. But this misleading conclusion is a consequence of the effective differences of the actual probe locations $\mathrm{y}^{+}$between the two experiments. Results have been calculated for the same runs as the earlier time series: $\mathrm{y}^{+} \approx 5.6,13.2$ and 22.3 for $-\mathrm{K}_{\mathrm{p}} \approx 0.008(\mathrm{lpg})$ and $\mathrm{y}^{+} \approx 7.4,16.0$ and 26.4 for $-\mathrm{K}_{\mathrm{p}} \approx 0.02(\mathrm{hpg})$. That is, the lpg measurement locations are closer to the wall than the corresponding hpg ones. Some differences between the two experiments may be explained by differences in $\mathrm{y}^{+}$relative to their nominal values. To account for this possibility, calculations for $\left\langle\mathrm{St}_{\mathrm{t}},{ }_{\mathrm{uy}}{ }^{+}\right\rangle$in the hpg experiment have been interpolated to the probe locations of the lpg experiment as done earlier to check $<\mathrm{u}^{+}>$. These interpolated values for hpg are plotted as solid curves in Figure 6a. In contrast to the $<\mathrm{u}^{+}>$distributions, interpolation of $<\mathrm{S}_{\mathrm{t}, \mathrm{uy}}{ }^{+}\left\{\mathrm{t}_{\mathrm{W}}{ }^{+}\right\}>$for the hpg cases to the same lpg positions gives some significantly different observations.

While there are differences in detail in Figure 6a, particularly the maximum values, the trends are approximately the same for both $<\mathrm{S}_{\mathrm{t}, \mathrm{uy}}{ }^{+}\left\{\mathrm{t}_{\mathrm{W}}{ }^{+}\right\}>$at each common location. The maxima and minima occur at about the same times $\mathrm{t}_{\mathrm{W}}{ }^{+}$for both pressure gradients. For the runs at $\mathrm{y}^{+} \approx 5.6$ and 13.2 the maxima and excursions from minimum to maximum are slightly greater for the hpg experiment than the lpg one. On average the hpg value of $<\mathrm{S}_{\mathrm{t}, \mathrm{uy}}{ }^{+}\left\{\mathrm{t}_{\mathrm{W}}{ }^{+}\right\}>$has a higher level than for the lpg at $\mathrm{y}^{+} \approx 5.6$ in contrast to the time-mean values of $\mathrm{S}_{\mathrm{t}}{ }^{+}$which are almost exactly the same at this location [McEligot et al., 2008a]. That is, the sweep passage causes more entropy generation in its vicinity for the hpg experiment. Away from the wall at $\mathrm{y}^{+} \approx 22$ the peak-to-peak oscillations of $<\mathrm{S}_{\mathrm{t}, \mathrm{uy}}{ }^{+}\left\{\mathrm{t}_{\mathrm{W}}{ }^{+}\right\}>$and its maximum value are much less for the hpg case - but the average level is higher over this range of $\mathrm{t}_{\mathrm{W}}{ }^{+}$ from deceleration to ejection to sweep to deceleration again for this case; at this location the DNS results of Spalart predict a slight reduction in the time-mean value of $\left(\mathrm{S}_{\mathrm{t}}\right)^{+}$as the pressure gradient is increased. The first minimum value of $<\mathrm{S}_{\mathrm{t}, \mathrm{uy}}{ }^{+}\left\{\mathrm{t}_{\mathrm{W}}{ }^{+}\right\}>$occurs near the maximum "instantaneous Reynolds shear stress" during the decelerating outflow phase ahead of the sweep; here the minimum $<\mathrm{S}_{\mathrm{t}, \mathrm{uy}}{ }^{+}\left\{\mathrm{t}_{\mathrm{W}}{ }^{+}\right\}>$is not as low for the hpg experiment as for lpg except in the measurements in the central part of the viscous layer near $\mathrm{y}^{+} \approx 13$ where these values are approximately the same. 
As seen in Figure 4, the gradient $<\partial \mathrm{u}^{+} / \partial \mathrm{t}^{+}>$at interface passage is slightly greater at $\mathrm{y}^{+} \approx 22(\operatorname{lpg})$ than for $\mathrm{y}^{+} \approx 26$ (hpg), corresponding to a slightly higher gradient $\partial \mathrm{u}^{+} / \partial \mathrm{y}^{+}$and - therefore - higher $<\mathrm{S}_{\mathrm{t}, \mathrm{uy}}{ }^{+}>$at that instant. The maximum value at $\Delta \mathrm{t}^{+} \approx 1.5$ later is also higher for this situation. While we concluded that $\left\langle\mathrm{u}^{+}>,<\mathrm{v}^{+}>\right.$and $\left\langle\mathrm{uv}>^{+}\right.$are generally broader in the hpg experiment than in the lpg one, one cannot make the same claim for the dominant entropy generation component (or the corresponding contribution to total dissipation). Though some coherent structures considered are apparently larger in wall coordinates, the timing of the transient variation of their entropy generation is not significantly affected.

In general, one may conclude that the dominant contribution $\left\langle\mathrm{S}_{\mathrm{t}, \mathrm{uy}}{ }^{+}{ }^{+}\right.$is much more sensitive to the distance from the wall than to an increase in favorable pressure gradient. The same qualitative observation can be made concerning the time-mean values of the direct and indirect (non-dimensional) entropy generation rates, as shown from DNS predictions for turbulent boundary layers [McEligot et al., 2008b].

\subsection{Implications for Turbulence Modeling}

For wall-bounded turbulent shear flows, the primary aim of turbulence modeling would be prediction of the skin friction coefficient or the pressure drop with a secondary aim being to provide an adequate description of the velocity profile for prediction of thermal and species boundary layers. It was recognized in the 1960s that the so-called "Universal Velocity Profile" and wall functions derived from it were not adequate for flows with significant pressure variation [Launder, 1963; Patel, 1965; McEligot, Ormand and Perkins, 1966]. (For internal flows an empirical correlation was reasonable for $\mathrm{c}_{\mathrm{f}}$ for $\mathrm{ReD}>2500$ or $-K_{p}<0.021$ [Reynolds, 1968].) This difficulty was cured with simple empirical modifications of mixing length treatments [McEligot, Ormand and Perkins, 1966; Huffman and Bradshaw, 1972] and with early k- $\varepsilon$ models [Jones and Launder, 1972].

The mean statistics of ME and the present temporal observations can give some further insight. For more complicated turbulence models, the difficulties seen for scaling event rates as the pressure gradient increases implies that, in the important viscous layer, predictions will be sensitive to the choice of scaling or multipliers for their adjustable constants/functions. (Proper description of the momentum equation should ensure a reasonable total stress distribution in the viscous layer.) ME data demonstrate that the Reynolds stress $\bar{u}^{2}$ does not vary significantly but $\bar{v}^{2}$ does in the outer region of the viscous layer. This observation may explain the success of the Durbin $v^{2}$-f model [Parneix, Durbin and Behnia, 1998] for some complex flows, such as strongly-heated gases with property variation [Hradisky et al., 2006 ]. Beyond the canonical flow assumptions of structure models [Panton, 2001; Bernard and Wallace, 2002] the various sub-grid-scale models of large eddy simulations may [Xu et al., 2004] or may not [Hradisky et al., 2006] successfully predict complex cases.

In the present temporal observations, at $\mathrm{y}^{+} \approx 15$ for hpg the "instantaneous Reynolds shear stress" and the maximum outward velocities have smaller peak values during ejections but the sizes are larger. Timing of significant events relative to sweep passage implies larger coherent structures at the higher pressure gradient. Timing and magnitude of the "instantaneous Reynolds shear stress" and, therefore, "instantaneous production" of tke per Diorio, Kelley and Wallace [2007] are modified by the pressure gradient. To provide reasonable predictions for non-canonical flows, turbulence models should account for these effects of pressure gradients, either directly or indirectly. For some applications, the change in the shape of sweep fronts might be significant. These variations can be expected to have effects on the development of boundary conditions for LES [Morrison and Westbury, 1996] and perhaps other advanced turbulence modeling. 
Morrison and Westbury interpreted their data as showing that small-scale turbulence is non-Gaussian and that this observation had consequences for LES where the sub-grid-scale motion is often modeled by an eddy-viscosity hypothesis. Thus, use of an eddy viscosity in LES is likely to cause problems if used unquestioningly. As is well known, near the wall $\mathrm{u}$ and $\mathrm{v}$ are non-Gaussian with the probability density

distributions of $\tilde{\mathrm{v}}$, and $(\tilde{\mathrm{u}} \tilde{\mathrm{v}})$ being affected by the pressure gradient [ME]. Consequently, sub-grid-scale models must account for the pressure gradient somehow in this region; a sufficiently fine grid, approaching DNS, in the viscous layer may treat this difficulty. Turbulence modeling is beyond the scope of this report (and its authors) so we invite sophisticated LES modelers to address this situation. 


\section{CONCLUDING REMARKS}

New fundamental measurements - for transient entropy generation rates and effects on coherent structures known as sweeps and ejections - have been obtained for turbulent flow in a converging duct, concentrating on the viscous layer where significant resistances to heat, momentum and mass transfer arise. A convergence induces a streamwise acceleration and, thereby, a favorable streamwise pressure gradient - a non-canonical situation. This study is the fourth in a sequence delving successively deeper into the structure of these flows. The general question being addressed is - how is the turbulence structure in the viscous layer modified by the favorable streamwise pressure gradient induced by such a convergence?

This study extends the earlier work of Eckelmann and colleagues with the same facility. The present objectives then are to determine whether streamwise mean pressure gradients affect the temporal behavior of entropy generation rates in the viscous layer and, if so, which aspects are affected significantly. Use of the oil channel permitted meaningful measurement of the wall-normal component and related statistics in the viscous layer where others could not obtain data due to problems of spatial resolution. Simultaneous time series data were obtained by McEligot and Eckelmann [2006] with an $\mathrm{X}$-probe and a wall sensor to determine $\tilde{\mathrm{u}}\{\mathrm{t}\}, \tilde{\mathrm{v}}\{\mathrm{t}\}$ and $\tilde{\tau_{\mathrm{W}}}\{\mathrm{t}\}$. Measurements with the X-probe at $\mathrm{y}^{+} \approx 5,7,10,15,25$ (nominal values) and the centerplane concentrated on four sets of conditions of increasing severity of non-dimensional pressure gradient $-\mathrm{K}_{\mathrm{p}}$ : two for converging flows and two for fully-developed ones. Of these, the present study examines the two extremes: (1) a high pressure gradient, nearing "laminarization," and (2), for comparison, a low pressure gradient corresponding to most earlier experiments and direct numerical simulations. Temporal behaviors of the coherent structures were deduced via quadrant splitting (QS), pattern recognition (PR) and Vita techniques. Sufficiently high thresholds were employed so that sweeps and abrupt ejections were detected. The PR and Vita approaches gave about the same results so the latter was employed for most of the observations presented. The Vita approach with $\widetilde{\mathrm{u}}\{\mathrm{t}\}$ is known to trigger on high values of $\partial \mathrm{u} / \partial \mathrm{t}$ corresponding to sweep passage and high values of the temporal entropy generation rate, so it is desirable to use it for the present objectives.

One might expect that, with constant values of the threshold multipliers, the measured turbulent structure rates might be compared across the viscous layer for the two extremes of pressure gradients. In terms of the non-dimensional event rates $\mathrm{f}^{+}$, the sweeps generally show reduced rates as the favorable pressure gradient is increased. This observation is consistent with those of Kline et al. and CMM. In contrast, the ejection rate (from quadrant splitting) increases with pressure gradient in the outer part of the viscous layer. However, selection of threshold criteria on other bases could modify these results and, possibly, change the trends observed; we have discussed some of the related considerations and qualitative explanations of some earlier observations. Whether these rates appear to decrease or increase with the pressure gradient depends on the quantity examined and the manner of sampling (and presentation). Investigators need to be cautious in their generalizations of this topic.

Overall, the trends and magnitudes of the measurements are approximately the same for hpg and lpg when presented in terms of wall coordinates. For both situations, the ensemble averages generally show first a Q2 deceleration continuing briefly $\left(\Delta \mathrm{t}^{+} \approx 2-4\right)$ as a Q3 wallward interaction as the Q4 sweep interface arrives. The main differences observable between the two pressure gradients for $\left\langle\mathrm{u}^{+}>,<\mathrm{v}^{+}>\right.$ and $<\mathrm{uv}^{+}$are (1) changes in the time lag between detections - representing modification of the shape of the sweep front and the sweep angle with the wall, (2) modification of the magnitude of the

"instantaneous Reynolds shear stress" $<\mathrm{uv}>^{+}$with wall distance and (3) broadening of the sweep fronts and ejections for the hpg experiment. 
Results new for even low pressure gradients are determinations of the temporal behavior of the dominant contribution to entropy generation. The local (pointwise) entropy generation rate per unit volume $\mathrm{S}^{\prime \prime}$ is a key to improving many energy processes and applications. The lowest entropy generation rate occurs during the Q2 deceleration phase and its maximum is slightly after interface passage. Typical sequences for the apparent bursting events, show a decrease in $<\mathrm{S}_{\mathrm{t}, \mathrm{uy}}{ }^{+}\left\{\mathrm{t}^{+}\right\}>$corresponding to the deceleration in $<\mathrm{u}^{+}>$as t$_{\mathrm{W}}{ }^{+}$increases, then a rapid increase with sweep/front passage, followed by a gradual recovery to/towards previous levels. Also the breadths of the peaks increase approaching the wall, indicating that the high temporal values of entropy generation rates in the vicinity of sweep fronts last for longer durations near the wall. The main increase in $\mathrm{S}_{\mathrm{t}}$,uy ${ }^{+}$at a probe location $\mathrm{y}^{+}$begins at values of $\mathrm{t}_{\mathrm{W}}{ }^{+}$ earlier than or ahead of the wall detection and near the front passage indicated by the earlier $\left\langle\mathrm{u}>^{+}\right.$results. For the runs at $\mathrm{y}^{+} \approx 5.6$ and 13.2 the maxima and excursions from minimum to maximum are slightly greater for the high pressure gradient experiment than the low pressure gradient one. Away from the wall at $\mathrm{y}^{+} \approx 22$ the peak-to-peak oscillations of $<\mathrm{S}_{\mathrm{t}, \mathrm{uy}}{ }^{+}\left\{\mathrm{t}_{\mathrm{W}}{ }^{+}\right\}>$and its maximum value are much less for the hpg case - but the average level is higher over the range of $\mathrm{t}_{\mathrm{W}}{ }^{+}$from deceleration to ejection to sweep passage to deceleration again for this case.

One may conclude that the dominant contribution $<\mathrm{St}_{\mathrm{t}, \mathrm{uy}}{ }^{+}\left\{\mathrm{t}^{+}\right\}>$is much more sensitive to the distance from the wall than to an increase in favorable pressure gradient. The levels of entropy generation rate decrease as $\mathrm{y}^{+}$increases; by the edge of the viscous layer, $<\mathrm{S}_{\mathrm{t}, \mathrm{uy}}{ }^{+}>$is small compared to the mean value of $\mathrm{St}_{\mathrm{t}}{ }^{+}$at the wall (predicted to be about 1.1 to 1.2). The same qualitative observation on transverse variation can be made concerning the time-mean values of the direct and indirect (non-dimensional) entropy generation rates, as shown from DNS predictions for turbulent boundary layers [McEligot et al., 2008b]. For the most part, one can generally say that the level of pressure gradient within the range from slight to near laminarizing does not cause significant change in mechanisms but there are differences in detail relating to timing, sizes and shapes of sweeps and ejections - and therefore related details for some turbulence models. 


\section{REFERENCES}

Adrian, R. J., 2007. Hairpin vortex organization in wall turbulence. Phys. Fluids, $\underline{19}, 041301$.

Adrian, R. J., C. D. Meinhart and C. D. Tomkins, 2000. Vortex organization in the outer region of the turbulent boundary layer. J. Fluid Mech., $\underline{422}$, pp. 1-54.

Alfredsson, P. H., and A. V. Johansson, 1984. On the detection of turbulence-generating events. J. Fluid Mech., 139 , pp. 325-345.

Alfredsson, P. H., A. V. Johansson, J. H. Haritonidis and H. Eckelmann, 1988. The fluctuating wall-shear stress and the velocity field in the viscous layer. Phys. Fluids, 31, pp. 1026-1033.

Andreopoulos, J., and J. H. Agui, 1996. Wall-vorticity flux dynamics in a two-dimensional turbulent boundary layer. J. Fluid Mech., $\underline{309}$, pp. 45-84.

Antonia, R. A., J. Kim and L. W. B. Browne, 1991. Some characteristics of small-scale turbulence in a turbulent duct flow. J. Fluid Mech., 233, pp. 369-388.

Aubertine, C. D., and J. K. Eaton, 2005. Turbulence development in a non-equilibrium turbulent boundary layer with mild adverse pressure gradient. J. Fluid Mech., 532, pp. 345-364.

Bejan, A., 1982. Entropy generation through heat and fluid flow. New York: Wiley.

Bernard, P. S., and J. M. Wallace, 2002. Turbulent flow. Analysis, measurement and prediction. Hoboken: Wiley.

Blackwelder, R. F., and H. Eckelmann, 1978. The spanwise structure of the bursting phenomenon. Lect. Notes Physics, 75, pp. 190-204.

Blackwelder, R. F., and J. H. Haritonidis, 1983. Scaling of the bursting frequency in turbulent boundary layers. J. Fluid Mech., 132, pp. 87-103.

Blackwelder, R. F., and R. E. Kaplan, 1976. On the wall structure of the turbulent boundary layer. J. Fluid Mech., $\underline{76}$, pp. 86-112.

Bogard, D. G., and W. G. Tiederman, 1986. Burst detection with single-point velocity measurements. J. Fluid Mech., 162 , pp. 389-413.

Bradshaw, P., 1974. Comments on 'Turbulent energy production, dissipation and transfer.' Phys. Fluids, 17, p. 2149.

Bradshaw, P., 1975. Bradshaw, P., 1975. An introduction to turbulence and its measurement, 2nd edition. Oxford: Pergamon.

Bradshaw, P., 1994. Turbulence: The chief outstanding difficulty of our subject. Exp. Fluids, $\underline{16}$, pp. 203-216.

Brandt, L., and D. S. Henningson, 2002. Transition of streamwise streaks in zero-pressure-gradient boundary layers. J. Fluid Mech., 472, pp. 229-261.

Brodkey, R. S., Y. G. Aouad, H. Valizadeh-Alavi and H. Eckelmann, 1985. Refinement of pattern recognition of coherent structures in turbulent shear flows and a comparison between detection techniques. Lec. Notes Physics, 235, pp. 279-291.

Brodkey, R. S., S. G. Nychas, J. L. Taraba and J. M. Wallace, 1973. Turbulent energy production, dissipation and transfer. Phys. Fluids, $\underline{16}$, pp. 2010-2011.

Brodkey, R. S., J. L. Taraba, S. G. Nychas and J. M. Wallace, 1974. Reply to comments by P. Bradshaw. Phys. Fluids, 17, p. 2150. 
Brodkey, R. S., J. M. Wallace and H. Eckelmann, 1974. Some properties of truncated turbulence signals in bounded shear flows. J. Fluid Mech., 63, pp. 209-224.

Chambers, F. W., H. D. Murphy and D. M. McEligot, 1983. Laterally converging flow. Part 2. Temporal wall shear stress. J. Fluid Mech., 127, pp. 403-428.

Clausius, R., 1887. Die mechanische Wärmetheorie. Braunschweig: Vieweg.

Corino, E. R., and R. S. Brodkey, 1969. A visual observation of the wall region in turbulent flow. J. Fluid Mech., 37, pp. 1-30.

Diorio, J., D. H. Kelley and J. M. Wallace, 2007. The spatial relationships between dissipation and production rates and vortical structures in turbulent boundary layers and mixing layers. Phys. Fluids, $\underline{19}$, No. 3, pp. 035101-1 to -8 .

Durst, F., J. Jovanovic and J. Sender, 1995. LDA measurements in the near-wall region of a turbulent pipe flow. J. Fluid Mech., 295, pp. 305-335.

Eckelmann, H., 1970. Experimentelle Untersuchungen in einer turbulenten Kanalströmung mit starken viskosen Wandschichten. Dissertation, Georg August Universität, Göttingen.

Eckelmann, H., 1974. The structure of the viscous sublayer and the adjacent wall region in a turbulent channel flow. J. Fluid Mech., 65, pp. 439-459.

Eckelmann, H., S. G. Nychas, R. S. Brodkey and J. M. Wallace, 1977. Vorticity and turbulence production in pattern recognized turbulent flow structures. Phys. Fluids Supplement, 20, pp. S225-S231.

Eckelmann, H., and M. Randolph, 1993. Measurements of spatial velocity gradients in a turbulent channel flow. Some New Trends on Fluid Mechanics and Theoretical Physics (Ed.: C. C. Lin and N. Hu), Beijing: Peking Univ. Press, pp. 358-361. (Conference was held in 1992.)

Eckelmann, H., and J. M. Wallace, 1981. A comparison of characteristic features of coherent turbulent structures found using the Variable Interval Time Average (VITA) technique and using the pattern recognition technique. Lec. Notes Physics, 136, pp. 292-303.

Eckelmann, H., J. M. Wallace and R. S. Brodkey, 1978. Pattern recognition, a means for detection of coherent structures in bounded turbulent shear flows. Proc., Dynamic Flow Conf., Baltimore, Md., pp. 161-172.

Finnicum, D. S., and T. J. Hanratty, 1988. Effect of favorable pressure gradients on turbulent boundary layers. A.I.Ch.E. Journal, $\underline{34}$, pp. 529-540.

Gersten, K., and H. Herwig, 1992. Strömungsmechanik. Braunschweig: Vieweg.

Goldstein, M. E., and S. J. Leib, 1993. Three-dimensional boundary-layer instability and separation induced by small-amplitude streamwise vorticity in the upstream flow. J. Fluid Mech., 246, pp. 21-41.

Guala, M., S. E. Hommema and R. J. Adrian, 2006, Large-scale and very-large-scale motions in turbulent pipe flow. J. Fluid Mech., 554, pp. 521-542.

Haas, S., and W. Schneider, 1996. Axisymmetric turbulent sink flows. IUTAM Symposium on Asymptotic Methods for Turbulent Shear Flows (Ed.: K. Gersten), Netherlands: Kluwer, pp. 81-94.

Honkan, A., and Y. Andreopoulos, 1997. Vorticity, strain-rate and dissipation characteristics in the nearwall region of turbulent boundary layers. J. Fluid Mech., 350, pp. 29-96.

Hradisky, M., T. Hauser, A. Richards and R. Spall, 2006. Large eddy simulation of strongly heated internal gas flows. AIAA paper 2006-3260. 
Huffman, G. D., and P. Bradshaw, 1972. A note on von Karman's constant in low Reynolds number turbulent flows. J. Fluid Mech., 53, pp. 45-60.

Jacobs, R. G., and P. A. Durbin, 2001. Simulations of bypass transition. J. Fluid Mech., 428, pp. 185-212.

Johansson, A. V., and P. H. Alfredsson, 1983. Effects of imperfect spatial resolution on measurements of wall-bounded turbulent shear flows. J. Fluid Mech., 137, pp. 409-421.

Johansson, A. V., P. H. Alfredsson and H. Eckelmann, 1987. On the evolution of shear-layer structures in near-wall turbulence. Advances in Turbulence (Ed.: G. Comte-Bellot and J. Mathieu), Berlin: Springer.

Johansson, A. V., P. H. Alfredsson and J. Kim, 1991. Evolution and dynamics of shear-layer structures in near-wall turbulence. J. Fluid Mech., 224, pp. 579-599.

Jones, M. B., I. Marusic and A. E. Perry, 2001. Evolution and structure of sink-flow boundary layers. J. Fluid Mech., $\underline{428}$, pp. 1-27.

Jones, W. P., and B. E. Launder, 1972. The prediction of laminarization with a two-equation model of turbulence. Int. J. Heat Mass Transfer, 15, pp. 301-314.

Jovanovic, J., and R. Hillerbrand, 2005. On peculiar property of the velocity fluctuations in wall-bounded flows. Thermal Sci., $\underline{9}$, No. 1, pp. 3-12.

Kays, W. M., 1966. Convective heat and mass transfer. New York: McGraw-Hill.

Kendall, J. M., 1998. Experiments on boundary layer receptivity to free-stream turbulence. AIAA paper 98-0530.

Kestin, J., 1980. Availability: the concept and associated terminology. Energy Int. J., $\underline{5}$, pp. 679-692.

Kim, J., P. Moin and R. D. Moser, 1987. Turbulent statistics in fully developed channel flow at low Reynolds number. J. Fluid Mech., 177, pp. 133-166.

Klebanoff, P. S., 1955. Characteristics of turbulence in a boundary layer with zero pressure gradient. NACA report 1247, pp. 1135-1153.

Kline, S. J., W. C. Reynolds, F. A. Schraub and P. W. Rundstadler, 1967. The structure of turbulent boundary layers. J. Fluid Mech., 30, pp. 741-773.

Kock, F., and H. Herwig, 2004. Local entropy production in turbulent shear flows: a high-Reynolds number model with wall functions. Int. J. Heat Mass Transfer, 47, pp. 2205-2215.

Kreplin, H.-P., and H. Eckelmann, 1979a. Instantaneous direction of the velocity vector in a fully-developed turbulent channel flow. Phys. Fluids, 22, pp. 1210-1211.

Kreplin, H.-P., and H. Eckelmann, 1979b. Propagation of perturbations in the viscous sublayer and adjacent wall region. J. Fluid Mech., 95, pp. 305-322.

Krogstad, P.-A., and P. E. Skare, 1995. Influence of a strong adverse pressure gradient on the turbulent structure in a boundary layer. Phys. Fluids, $\underline{7}$, pp. 2014-2024.

Laadhari, F., 2007. Reynolds number effect on the dissipation function in wall-bounded flows. Phys. Fluids, 19, No. 3, pp. 038101-1 to -4.

Landahl, M. T., 1980. A note on an algebraic instability of inviscid parallel shear flows. J. Fluid Mech., 98, pp. 243-251.

Launder, B. E., 1963. The turbulent boundary layer in a strongly negative pressure gradient. Master's thesis, MIT. Also MIT Gas Turbine Lab. Rpt. 71. 
Launder, B. E., 1964. Laminarization of the turbulent boundary layer by acceleration. Sc.D. thesis, MIT. Also MIT Gas Turbine Lab. Rpt. 77 and NASA N66-16042.

Liepmann, H. W., 1943. Investigations on laminar boundary-layer stability and transition on curved boundaries. NACA Wartime Report W-107, ACR No. 3H30.

Liu, Z., R. J. Adrian and T. J. Hanratty, 2001. Large-scale modes of turbulent channel flow: transport and structure. J. Fluid Mech., $\underline{448}$, pp. 53-80.

Lu, S. S., and W. W. Willmarth, 1973. Measurements of the structure of the Reynolds stress in a turbulent boundary layer. J. Fluid Mech., $\underline{60}$, pp. 481-511.

Luchik, T. S., and W. G. Tiederman, 1987. Timescale and structure of ejections and bursts in turbulent channel flows. J. Fluid Mech., 174, pp. 529-552.

Mayle, R. E., 1991. The role of laminar-turbulent transition in gas turbine engines. J. Turbomachinery, $\underline{113}$, pp. 509-537.

McEligot, D. M., and H. Eckelmann, 2003. Effects of laterally converging flows on mean turbulence structure in the viscous layer. Tech. rpt. INEEL/EXT-2002-697, Idaho National Engineering and Environmental Laboratory.

McEligot, D. M., and H. Eckelmann, 2006. Laterally converging duct flows: Part 3. Mean turbulence structure in the viscous layer. J. Fluid Mech., 549, pp. 25-59.

McEligot, D. M., K. P. Nolan, E. J. Walsh and E. Laurien, 2008a. Effects of pressure gradients on entropy generation in the viscous layers of turbulent wall flows. Int. J. Heat Mass Transfer 51, pp. 1104-1114.

McEligot, D. M., L. W. Ormand and H. C. Perkins, 1966. Internal low Reynolds number turbulent and transitional gas flow with heat transfer. J. Heat Transfer, $\underline{88}$, pp. 239-245.

McEligot, D. M., E. J. Walsh, E. Laurien and P. R. Spalart, 2008b. Entropy generation in the viscous parts of turbulent boundary layers. J. Fluids Eng., 130, pp. 061205-1 to -12.

Moin, P., and J. Kim, 1982. Numerical investigations of turbulent channel flow. J. Fluid Mech., 118, pp. 341-377.

Morrison, J. F., 2007. The interaction between inner and outer regions of turbulent wall-bounded flow. Phil. Trans. R. Soc. A, $\underline{365}$, pp. 683-698.

Morrison, J. F., and P. Bradshaw, 1989. Bursts and wall shear stress in turbulent boundary layers. Proc., 7th Symp. Turb. Shear Flows, Stanford, paper 2-2.

Morrison, J. F., C. S. Subramanian and P. Bradshaw, 1992. Bursts and the law of the wall in turbulent boundary layers. J. Fluid Mech., 241, pp. 75-108.

Morrison, J. F., H. M. Tsai and P. Bradshaw, 1989. Conditional-sampling schemes for turbulent flow based on the variable-interval time averaging (VITA) algorithm. Exp. Fluids, 7, pp. 173-189.

Morrison, J. F., and P. S. Westbury, 1996. Perspectives on wall turbulence: The use of complementary techniques. Exp. Th. Fluid Sci., 13, pp. 211-222.

Murphy, H. D., F. W. Chambers and D. M. McEligot, 1983. Laterally converging flow. Part 1. Mean flow. J. Fluid Mech., 127, pp. 379-401.

Narasimha, R., and K. R. Sreenivasan, 1979. Relaminarization of fluid flows. Adv. Appl. Mech., 19 , pp. 221-309.

Neumann, S. O., J. von Wolfersdorf and B. Weigand, 2005. A numerical study of entropy production rates in a film cooling configuration. GAMM (Gesellschaft für Angewandte Mathematik und Mechanik e.V.) 
Nychas, S. G., H. C. Hershey and R. S. Brodkey, 1973. A visual study of turbulent shear flow. J. Fluid Mech., $\underline{61}$, pp. 513-540

Panton, R. L., (Ed.), 1997. Self-sustaining mechanisms of wall turbulence. Southampton: Computational Mechanics Pubs.

Panton, R. L., 2001. Overview of the self-sustaining mechanisms of wall turbulence. Prog. Aero. Sci., $\underline{37}$, pp. 341-383.

Panton, R. L., 2005. Review of wall turbulence as described by composite expansions. Appl. Mech. Rev., $\underline{58}$, pp. 1-36.

Panton, R. L., 2007. Composite asymptotic expansions and scaling wall turbulence. Phil. Trans. R. Soc. $A, \underline{365}$, pp. $733-754$.

Panton, R. L., 2008. Personal electronic communication. U. Texas, 26 February.

Parneix, S., P. Durbin and M. Behnia, 1998. Computation of 3D turbulent boundary layers using the $\mathrm{v}^{2}$-f model. Flow, Turbulence and Combustion, 10, pp. 19-46.

Patel, V. C., 1965. Calibration of the Preston tube and limitations on its use in pressure gradients. J. Fluid Mech., 23, pp. 185-208.

Randolph, M., H. Eckelmann and S. G. Nychas, 1987. Identification of sweeps with the help of the instantaneous velocity gradient dU/dy. Advances in Turbulence, Berlin: Springer, pp. 408-415.

Reynolds, H. C., 1968. Internal low Reynolds number turbulent heat transfer. Ph. D. thesis, Aero. Mech. Engr., Univ. Arizona. Also DDC AD 669254.

Robinson, S. K., 1991. Coherent motions in the turbulent boundary layer. Annu. Rev. Fluid Mech., 23, pp. 601-639.

Rotta, J. C., 1962. Turbulent boundary layers in incompressible flow. Progress in Aeronautical Sciences, Vol. 2, pp. 1-219, Oxford: Pergamon Press.

Schlichting, H., 1982. Grenzschicht-Theorie, Achte Auflage. Karlsruhe: G. Braun.

Senecal, V. E., 1952. Fluid flow in the transition zone. Ph.D. thesis, Carnegie Inst. Tech.

Spalart, P. R., 1986. Numerical study of sink-flow boundary layers. J. Fluid Mech., 172, pp. 307-328.

Spalart, P. R., 1988. Direct simulation of a turbulent boundary layer up to $\operatorname{Re} \theta=1410$. J. Fluid Mech., 187, pp. 61-98.

Theodorsen, T., 1952. Mechanism of turbulence. Proc., 2nd Midwestern Conf. on Fluid Mechanics, Ohio State Univ.

Tomkins, C. D., and R. J. Adrian, 2003. Spanwise structure and scale growth in turbulent boundary layers. J. Fluid Mech., $\underline{490}$, pp. 37-74.

Townsend, A. A., 1956. The structure of turbulent shear flow. Cambridge: Univ. Press.

Townsend, A. A., 1961. Equilibrium layers and wall turbulence. J. Fluid Mech., 11, pp. 97-120.

Wallace, J. M., and R. S. Brodkey, 1977. Reynolds stress and joint probability density distributions in the u-v plane of a turbulent channel flow. Phys. Fluids, 20, pp. 351-355.

Wallace, J. M., R. S. Brodkey and H. Eckelmann, 1977. Pattern-recognized structures in bounded turbulent shear flows. J. Fluid Mech., 83, pp. 673-693.

Wallace, J. M., H. Eckelmann and R. S. Brodkey, 1972. The wall region in turbulent shear flows. J. Fluid Mech., 54, pp. 39-48. 
Wallace, J. M., and J. F. Foss, 1995. The measurement of vorticity in turbulent flows. Annu. Rev. Fluid Mech., 27, pp. 469-514.

Wilhelm, D., C. Härtel and H. Eckelmann, 1998. On the relation between fronts and high-shear layers in wall turbulence. Flow, Turb. Comb., $\underline{60}$, pp. 87-103.

Willmarth, W. W., and S. S. Lu, 1972. Structure of the Reynolds stress near the wall. J. Fluid Mech., 55, pp. 65-92.

Wu, X., R. G. Jacobs, J. C. R. Hunt and P. A. Durbin, 1999. Simulation of boundary layer transition induced by periodically passing wakes. J. Fluid Mech., $\underline{398}$, pp. 109-153.

Wundrow, D. W., and M. E. Goldstein, 2001. Effect on a laminar boundary layer of small-scale streamwise vorticity in the upstream flow. J. Fluid Mech., $\underline{426}$, pp. 229-262.

Xu, X., J. S. Lee, R. H. Pletcher, A. M. Shehata and D. M. McEligot, 2004. Large eddy simulation of turbulent forced gas flows in vertical pipes with high heat transfer rates. Int. J. Heat Mass Transfer, 47, pp. 4113-4123. 